Check for updates

Cite this: Mater. Chem. Front., 2020, 4, 3130

\section{Recent progress on PEDOT:PSS based polymer blends and composites for flexible electronics and thermoelectric devices}

\author{
Yan Yang, Hua Deng (D) * and Qiang Fu (D)
}

With the development of flexible electronic devices such as organic electrodes, medical sensors and light-emitting diodes, the conductive polymer, poly(3,4-ethylenedioxythiophene):poly(styrenesulfonate) (PEDOT:PSS), has attracted tremendous research attention due to its unique properties, such as flexibility, high electrical conductivity, promising thermoelectric properties and good water processability. Over the past few years, PEDOT:PSS has been extensively investigated and many research studies have been devoted to optimizing these versatile properties via molecular or structural design. Incorporating various polymers and fillers into PEDOT:PSS through blending and mixing is the most commonly used method to modify different properties of such conductive polymers, but a related review paper is still missing, especially on stretchable electronics and thermoelectric applications. Thus, this paper reviews recent progress on PEDOT:PSS based blends and composites used in the above two applications fields. Recent studies are divided into studies on miscible polymer blends, immiscible polymer blends, inorganic filler/polymer composites and carbon filler/polymer composites. The main focus is on the electrical conductivity, stretchability and thermoelectric properties of these blends and composites based on PEDOT:PSS. It is demonstrated that careful attention needs to be paid on the structure of these blends and composites to achieve desired properties. In these PEDOT:PSS based blends and composites, the distribution of polymers and fillers within PEDOT:PSS, and the composition and configuration of PEDOT:PSS itself are governed by the interfacial interaction, processing and posttreatment methods. It is widely demonstrated that various properties, such as electrical, thermoelectric and mechanical properties, of PEDOT:PSS blends and composites could be systematically adjusted through careful design of the above aspects in these materials. Nevertheless, there are still a number of issues that need to be further investigated to expand the application of PEDOT:PSS based materials, such as constructing hierarchical structures or nano confined PEDOT:PSS in an elastic matrix to form conductive pathways with a better balance between electrical conductivity and stretchability; filler distribution, interfacial interaction and orientation of the filler should be taken into account to optimize the thermoelectric properties of PEDOT:PSS based composites; self-powered flexible wearable electronics using PEDOT:PSS composites or blends based on the Seebeck effect is still in its infancy and needs much more attention.
Received 9th May 2020,

Accepted 24th July 2020

DOI: $10.1039 / \mathrm{d} 0 \mathrm{qm} 00308 \mathrm{e}$

rsc.li/frontiers-materials
Flexible electronic materials like finely structurally designed metal wires or films, ${ }^{1}$ carbon nanotubes ${ }^{2,3} /$ graphene $^{4,5}$ and its composites, ${ }^{6-8}$ and conducting polymers ${ }^{9,10}$ are investigated to serve as flexible electrodes, light-emitting diodes, perovskite solar cells, health monitoring sensors and thermoelectric devices. Among them, conductive polymers are one of the most promising candidates because of their prominent advantages of unique flexibility, high intrinsic electrical conductivity, low density and ease of processing. Since the discovery of the first conductive polymer polyacetylene (PA) in $1977,{ }^{11}$ the design and application of conductive polymers have attracted much attention. In addition to the linear structure of conductive
College of Polymer Science and Engineering, State Key Laboratory of Polymer Materials Engineering, Sichuan University, Chengdu 610065, China.

E-mail: huadeng@scu.edu.cn 
polymers, conductive polymers with isopropyl rings such as polypyrrole (PPy), ${ }^{12,13}$ polyaniline (PANi), ${ }^{14,15}$ and polythiophene and its derivatives ${ }^{16}$ have also been developed. Processability is a major challenge for intrinsically conductive polymers. Due to the rigidity of the conjugate skeleton and the stacking force between the molecules, most conductive polymers are difficult to dissolve and degrade at temperatures below their melting point.

Poly(3,4-ethylenedioxythiophene):poly(styrenesulfonate) (PEDOT: PSS) is a polyelectrolyte consisting of positively charged electrically conducting conjugated PEDOT and negatively charged insulating PSS. PSS polymer anions can stabilize conjugated polymer cations in water and some polar organic solvents. ${ }^{17}$ Such a method is the most significant technical breakthrough in improving the processability of

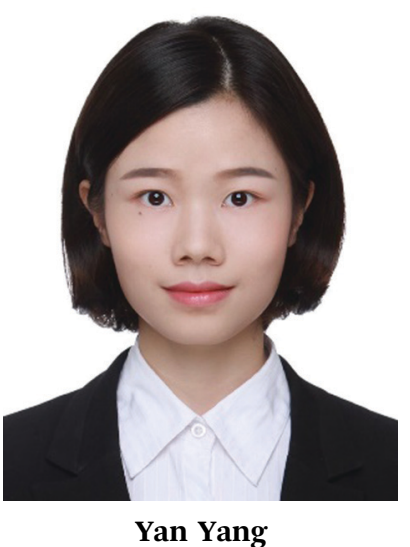

Yan Yang is currently pursuing her Master's degree at the College of Polymer Science and Engineering, Sichuan University. Her research interest includes organic thermoelectric materials for flexible electronics and selfpowered sensors. Her other research interests include dielectric polymer composites for high energy density materials. intrinsically conductive polymers. Currently, PEDOT:PSS aqueous is a commercially available product with different electrical properties, such as Clevios PH500, PH510, PH1000, Clevios PVP AI 4083 and Clevios PVP CH8000. Relevant product information of different PEDOT:PSS aqueous are listed in Table 1. PH1000 is the commonly used one in lab-scale research. The chemical structure and morphology of PEDOT:PSS are demonstrated in Fig. 1. It consists of positively charged conductive conjugated PEDOT and negatively charged insulating PSS. The insoluble PEDOT short chain is adherent to the water-soluble PSS long molecular chain to form a grain by coulomb force, so that it can be steadily dispersed in water. A grain with a diameter of $30-50 \mathrm{~nm}$ is composed of numerous tangles, and each tangle is made of a single PSS chain with several PEDOT segments. Ultimately, the morphology of the dispersion is generally described as being composed of a PEDOT-enriched core and a polyanion (PSS ${ }^{-}$) shell ${ }^{18,19}$ (Fig. 1a and b). Deposited gel particles of PEDOT:PSS thus result in a pancake-like morphology ${ }^{20-22}$ due to the lateral shrinkage and thickness reduction during drying (Fig. 1c). PEDOT chains may interact with each other through $\pi$-orbital interactions in the core region. ${ }^{23-25}$ And hydrogen bonds are formed based on $\mathrm{HSO}_{3}$ groups between the PSS shells, which provides cohesive strength to materials (Fig. 1d). Various processing methods, secondary doping and post treatment have been verified to lead to improvement in electrical conductivity by removing the excess doped phase (insulating PSS), leading to phase separation or inducing morphological re-arrangement. Polar solvents (such as DMSO, ethylene glycol, co-solvents, etc.), ${ }^{26}$ some acids (chloroplatinic acid, ${ }^{27}$ sulfonic acid, ${ }^{28}$ mineral acid $^{29}$ ) or ionic liquids can largely increase the conductivity of PEDOT:PSS films. ${ }^{30}$

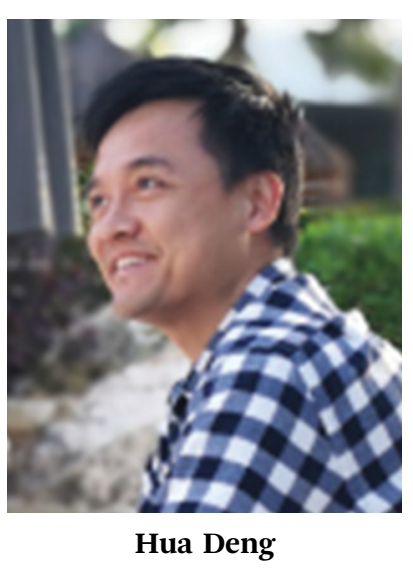

Hua Deng is currently a full-time professor at the College of Polymer Science and Engineering, Sichuan University. He obtained his $P h D$ in 2008 from Queen Mary University of London under the supervision of Professor Ton Peijs. From 2008 to 2009, he worked for Nanocyl S.A. R\&D department. In 2009, he joined Sichuan University as a lecturer and became an Associate Professor and a Full Professor in 2011 and 2015, respectively. $\mathrm{He}$ is the associate editor of Nanocomposites (Taylor \& Francis), and an editorial board member and guest editor of Composites Communications. He is a member of the Standing Committee of the China Composite Materials Society for dielectric polymer composites and applications, a member of the thermal conductive composites committee, and a member of the nanocomposite committee. His main research interest is in the processing of functional polymer composites, including conductive polymer composites, dielectric polymer composites, strain/pressure sensing polymer composites, etc.

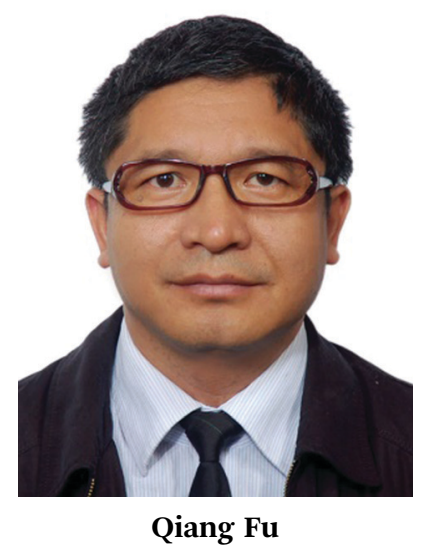

Qiang Fu obtained his PhD (1993) from Sichuan University in China. Currently, he is the Dean and a professor at the College of Polymer Science and Engineering, Sichuan University. From 1995 to 1997, he worked as a postdoctoral follow in Prof. Stephen Z. D. Cheng's research group at the University of Akron, USA. At present, he is the Dean of the College of Polymer Science and Engineering, Sichuan University, the vice president of China Plastics Processing Association, and a member of the polymer Professional Committee of China Chemical Society. He also serves as the Editor of Polymer (Elsevier). His research interests are focused on high-performance and multifunctionality of polymer blends and polymer nanocomposites, and processingstructure/morphology-performance relationships of polymer materials. 
Table 1 Product information of different levels of PEDOT:PSS aqueous

\begin{tabular}{llllll}
\hline Name & Ratio (PEDOT: PSS) & Solid content (wt\%) & Conductivity $\left(\mathrm{S} \mathrm{cm}^{-1}\right)$ & Particle size (nm) & Applications \\
\hline Clevios PVP CH8000 & $1: 20$ & $2.5-3.0$ & $10^{-5}-10^{-6}$ & 25 & $\begin{array}{l}\text { Organic electronic material } \\
\text { Cavity injection layer }\end{array}$ \\
Clevios PVP AI 4083 & $1: 6$ & $1.3-1.7$ & $10^{-3}-10^{-4}$ & 40 & $\begin{array}{l}\text { Organic electronic material } \\
\text { Optical electronic devices }\end{array}$ \\
Clevios P & $1: 2.5$ & & & Electrode materials \\
& & $1.2-1.4$ & $0.2-1$ & 80 & Antistatic layer \\
Clevios PH 500 & $1: 2.5$ & $1.0-1.4$ & $0.2-1$ & 30 & Electrode materials \\
Clevios PH 510 & $1: 25$ & $1.5-1.9$ & $0.2-1$ & 30 & Electrode materials \\
Clevios PH1000 & $1: 2.5$ & $1.0-1.3$ & $0.2-1$ & 30 & Electrode materials
\end{tabular}

(a)
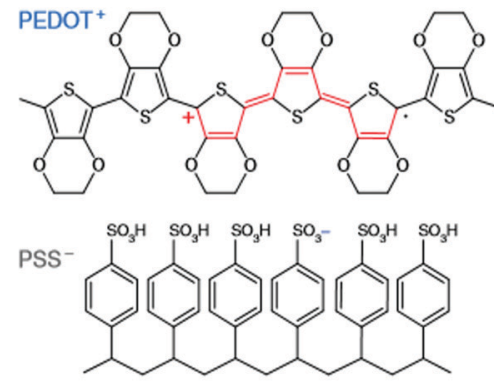

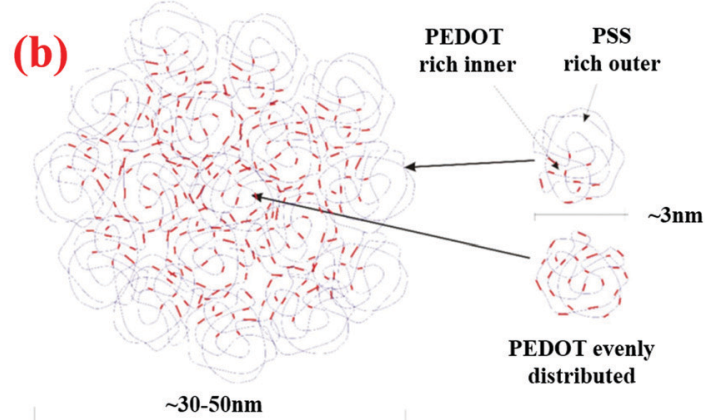

(c)
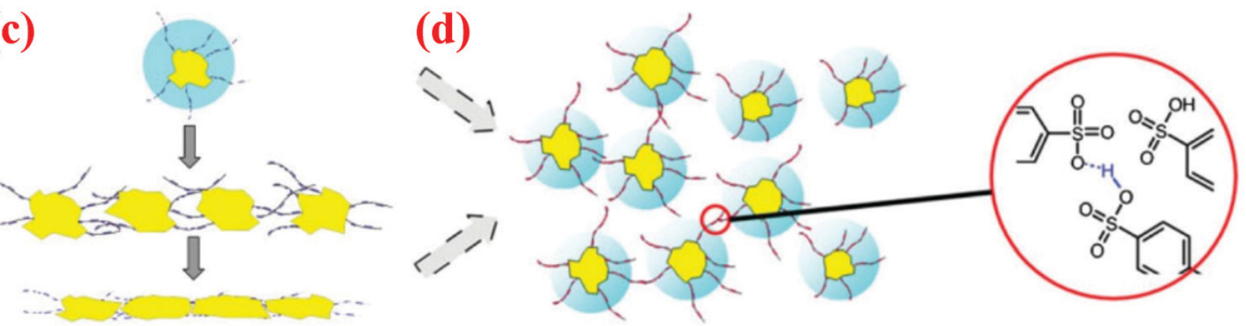

Fig. 1 (a) The chemical structure of PEDOT:PSS; (b) hypothesis for the morphology of individual PEDOT:PSS grains; model of PEDOT:PSS solid thin film formation on a substrate. (c) Side views of the model for a pancake-like morphology of PEDOT:PSS; (d) it is assumed that hydrogen bonds are developed between $\mathrm{HSO}_{3}$ groups of the PSS-rich outer shell of individual gel particles and therefore promote adhesion between individual PEDOT:PSS grains. Produced with permission, ${ }^{22}$ Copyright (C2016, Springer Nature. ${ }^{21}$ Copyright $(C) 2009$ Wiley-VCH Verlag GmbH \& Co. KGaA, Weinheim.

For instance, an extremely high conductivity of $4380 \mathrm{~S} \mathrm{~cm}^{-1}$ has been observed through a post treatment with $\mathrm{H}_{2} \mathrm{SO}_{4}$, compared to about $1 \mathrm{~S} \mathrm{~cm}^{-1}$ without any secondary dopant. ${ }^{31}$ There have been some reviews that reported the methods and suggested mechanisms for enhancement of its conductivity. ${ }^{32-34}$ In addition to its excellent electrical conductivity, its superior transparency $(80-95 \%)$ in the visible range and satisfactory flexibility make it a good candidate to replace brittle and high cost indium tin oxide (ITO) as a transparent electrode in optoelectronic devices. And several research groups have reviewed its salient properties in optoelectronic devices from different viewpoints of applications such as organic and perovskite solar cells, ${ }^{35-37}$ photoelectron spectroscopy, ${ }^{38}$ organic light-emitting diodes ${ }^{39}$ and electrode materials for supercapacitors. ${ }^{40-42}$ Moreover, PEDOT:PSS is a promising organic thermoelectric material due to its advantages of good thermoelectric properties $(Z T \sim 0.42)$, ultra-low thermal conductivity, processability, non-toxicity and unique flexibility, compared with traditional inorganic thermoelectric materials.
The methods and mechanisms for the enhancement of thermoelectric properties of neat PEDOT:PSS and its potential applications have also been outlined in the literature. ${ }^{43-45}$

Despite the high electrical conductivity, low sheet resistance, superior transparency, and thermoelectric properties of PEDOT: PSS, it is still in the development stage especially for applications as conductive, flexible or even stretchable, wearable electronic devices. The as-cast PEDOT:PSS films demonstrate an inherent tensile strain of around $2 \%$. The next generation of electronic devices, like skin electronics, requires materials possessing both electrical conductivity and deformability beyond flexibility, i.e., stretchability (elongation $>10 \%{ }^{46-48}$ ). In recent years, there have been vast efforts to establish stretchable materials based on PEDOT:PSS. Based on many research studies and review papers ${ }^{49,50}$ there are generally two main routes to prepare stretchable PEDOT:PSS elastic conductors. The first route is based on geometric engineering, which is similar to the approaches associated with inorganic stretchable electronic 
materials. ${ }^{51,52}$ A thin layer, ${ }^{53}$ wavy pattern, ${ }^{54-57}$ buckled $^{58-61}$ and wrinkled $^{62-65}$ conductive polymer network is deposited on the surface of an elastomer or constructed inside an elastic substrate to convert the overall tensile strain into local bending strain. However, the failure of electronic devices with such a multilayer structure mostly originates from the debonding between different layers because of poor interfacial interactions and large mechanical mismatch. It is urgent to develop a fully organic and intrinsically stretchable conductor. Besides, the thermoelectric properties (Seebeck coefficient and electrical conductivity) are not superior to those of traditional inorganic semiconductors. It is desired to combine the flexibility and solution processability of polymers with the thermoelectric properties of inorganic thermoelectric materials.

The second method is modification, blending and compounding of PEDOT:PSS. And the structure and morphology of the composites are adjusted and controlled to optimize various properties. Typically, the preparation of composites is an effective way to incorporate different functions into one system. Through regulating the proportion, distribution, combination, interaction and structure of the materials with different components, excellent comprehensive performance could be obtained. Among various approaches to prepare polymeric composites, blending and mixing are the most commonly used methods in experiment research, plant trial and mass production. Bulk processing (more than tens of microns thick) is needed to fulfill current flexible electronics design requirements. However, there are two main obstacles for preparing bulk materials for PEDOT:PSS. Firstly, both PEDOT and PSS are semi-crystalline polymers with no observable transmittance temperature and melting temperature. Therefore, it is difficult to process or modify PEDOT:PSS by a traditional melting process. Secondly, PEDOT:PSS can only be dissolved in water and cannot be re-dispersed once dried. Solution blending or mixing is the most effective strategy to prepare PEDOT:PSS based blends and composites. It is found that the solid content of PEDOT:PSS aqueous is very low (Table 1), which hinders the processing conditions. However, review papers on such topics are still scarce, especially on PEDOT:PSS based polymer blends and composites for stretchable electronics and thermoelectric applications.

In this review, we summarized the recent progress on PEDOT:PSS based blends and composites containing different components. To better assess the relationship between the structure and properties of these blends, we mainly focused on the effects of different compatibility and distribution states on the tensile and conductive properties of these materials. For PEDOT:PSS composites containing inorganic and carbon fillers, we focused on discussing their electrical and thermoelectric properties. Most of the current reviews on PEDOT:PSS are from the perspective of a specific property or application, such as its applications in organic solar cells, dye-sensitized solar cells, supercapacitors, fuel cells, thermoelectric devices and stretchable devices. ${ }^{65,66}$ Very few of them illustrated the effects of composite composition, interactions, and structure on the properties of PEDOT:PSS based blends and composites.
Therefore, this review should be able to serve as a complementary paper to the review papers in the literature to provide a better picture of the overall progress on PEDOT:PSS related materials and applications.

\section{Stretchable conductive PEDOT:PSS blends and composites}

Although PEDOT:PSS films are considered as promising candidates for flexible organic light emitting diodes, transparent electrodes, and electrostatic coatings, most of the applications depend not only on their electrical properties, but also on their mechanical properties. Their mechanical properties and related influencing factors have been systematically investigated by several research groups. Lang and coworkers did pioneering research on the microstructure ${ }^{21}$ and mechanical properties $^{67}$ of PEDOT:PSS films. From characterization with a micro tensile test setup, the authors observed that these films exhibit Young's modulus between 1-2.7 GPa, tensile strength between 25-55 MPa, and strain at fracture between 3-5\%. Most importantly, all these properties depend on the relative humidity of the environment. In their subsequent research, ${ }^{68}$ the effect of humidity on mechanical properties was attributed to the variation in the strength of hydrogen bonds between PSS shells. At low relative humidity, these hydrogen bonds are very strong and a trans-granular fracture occurs under tensile load, that is, a brittle fracture occurs through a single grain. However, higher relative humidity leads to hydrophilic PSS shells adsorbing water, resulting in expansion and weakened hydrogen bonds. In tensile tests, individual grains may even slide towards each other, leading to an inter granular plastic fracture. Besides humidity, ${ }^{69}$ other factors such as the temperature, ${ }^{70}$ tensile strain rate, ${ }^{71}$ and ratio between PEDOT and PSS $^{72}$ should also be taken into account when measuring the mechanical properties of PEDOT:PSS. Nevertheless, the brittleness of PEDOT:PSS limits their application in flexible electronic equipment. To address this issue, incorporating stretchable polymers or small molecules into PEDOT:PSS is thought to be an effective and efficient strategy to enhance the overall stretchability. Due to the degree of miscibility between PEDOT:PSS and added extra components, they are divided into miscible and immiscible polymer blends. Therefore, the following sections are organized regarding the structure and properties of miscible and immiscible PEDOT:PSS blends, respectively.

\subsection{Molecular level miscible polymer blends}

Small molecular plasticizers, such as surfactants (Zonyl, ${ }^{73}$ xylitol $^{74}$ Triton $\mathrm{X}-100^{75}$ and so on ref. 76 and 77) and co-solvents could improve the processability or the work function in specific applications of PEDOT:PSS, and they may also significantly alter the mechanical properties. For instance, deposited PEDOT:PSS films containing increasing concentration of Zonyl fluorosurfactant exhibit a qualitative increase in the size of PEDOT-rich domains, visible in the phase-contrast images (Fig. 2a). A sample containing $10 \mathrm{wt} \%$ Zonyl exhibits rather high aspect ratio fibrils, which is conducive to good compliance and ductility. ${ }^{76}$ 

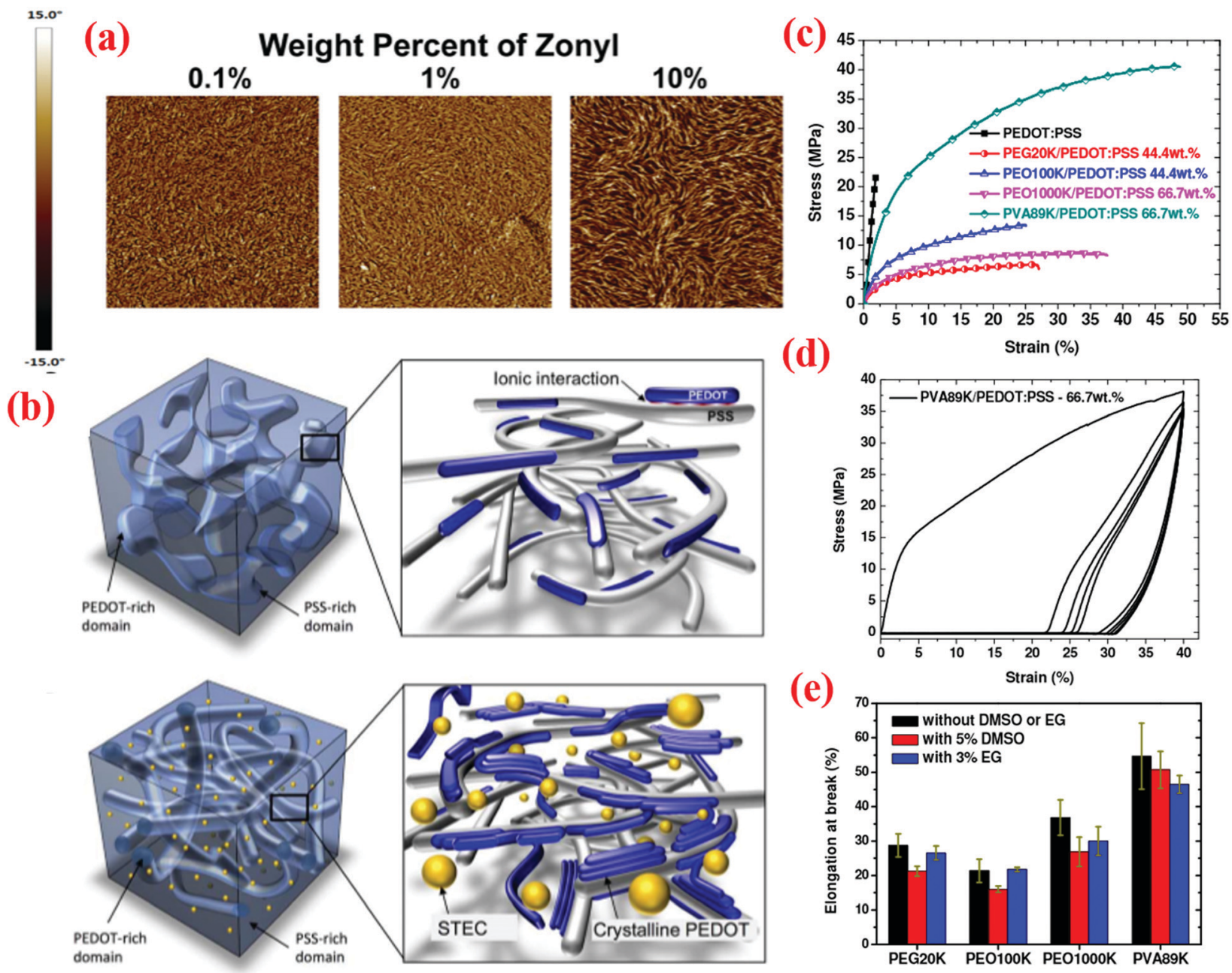

(d)

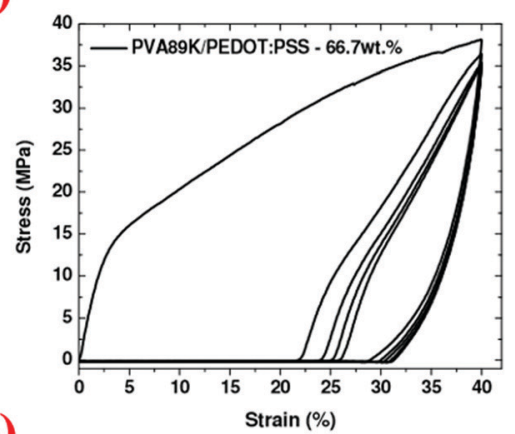

(e)

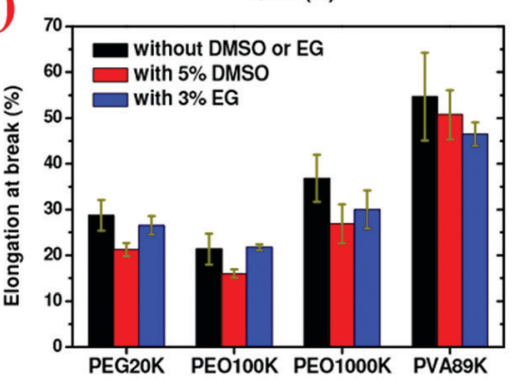

Fig. 2 (a) AFM images of PEDOT:PSS films with different contents of Zonyl additives. The dimensions of each AFM image are $1.5 \mu$ m $\times 1.5 \mu$ m; (b) schematic diagram representing the morphology of PEDOT:PSS with a STEC enhancer; (c) stress-strain curves of polymer blends containing PVA, PEO and PEG; (d) stress-strain curves of PVA89k/PEDOT:PSS-66.7 wt\%; (e) the elongation of polymer blends with or without DMSO and EG treatment. Produced with permission, ${ }^{76}$ Copyright (C) 2014 Wiley-VCH Verlag GmbH \& Co. KGaA, Weinheim. ${ }^{57}$ Copyright (C) 2017, The Authors. ${ }^{82}$ Copyright (C) 2015 American Chemical Society.

Mixing with a plasticizer allows PEDOT:PSS films to partially swell, weakening the hydrogen bonds between PSS molecular chains, and increase the free volume of twisted PEDOT and PSS molecular chains, so that the chain segment can be fully relaxed to withstand mechanical damage and tensile strain. Meanwhile, small molecular additives can increase the mobility of charge carriers between colloidal particles thus achieving higher electrical conductivity. Although the use of additives can increase the tensile strain up to $20-50 \%$, an unavoidable disadvantage is that they can easily seep out during subsequent application, which could lead to degradation of various properties. Other than the above small molecule plasticizers, ionic liquid doping is another way to obtain highly stretchable PEDOT:PSS films. ${ }^{78-81}$ Bao and co-workers ${ }^{57}$ reported a highly stretchable conducting polymer by incorporating ionic additives, with conductivity over $3100 \mathrm{~S} \mathrm{~cm}^{-1}$ under $0 \%$ strain and over $4100 \mathrm{~S} \mathrm{~cm}^{-1}$ under $100 \%$ strain when supported on styrene ethylene butylene styrene (SEBS) or $600 \mathrm{~S} \mathrm{~cm}^{-1}$ as freestanding films. The author proposed that sulfonate or sulfonimide anion ionic liquids can weaken the electrostatic interaction between PEDOT and PSS, and trigger PEDOT to partially aggregate forming "hard" conductive networks inside a soft PSS matrix as shown in Fig. $2 b$.

Motivated by advantages including low cost and large-scale and continuous production, blending PEDOT:PSS with a high loading of soft polymers is a promising method to achieve stretchable electronic materials. As PEDOT is an aqueous solution which is hardly dissolvable in organic solvents, blending PEDOT:PSS aqueous with water-soluble polymers like PVA, ${ }^{82-85}$ $\mathrm{PEO}^{82,83,86,87}$ and $\mathrm{PEG}^{82}$ has been extensively studied. For instance, the electrical conductivity and stretchability are dependent on the morphology and crystallinity presented by the insulating soft polymer according to Reynolds and co-workers. ${ }^{83}$ Compared to blending with an insulating amorphous soft polymer (PEO), crystalline soft polymer blends show a more rapid increase in conductivity. This is mainly due to the crystallization driven exclusion of the conducting polymer into 
Table 2 Brief summary of current PEDOT:PSS stretchable blends

\begin{tabular}{|c|c|c|c|c|c|c|}
\hline No. & Preparation & $\begin{array}{l}\text { PEDOT:PSS } \\
\text { content }\end{array}$ & $\begin{array}{l}\text { Conductivity or } \\
\text { sheet resistance }\end{array}$ & Elongation & $\begin{array}{l}\text { Resistance change } \\
\left(R / R_{0} \text {-strain }\right)^{a}\end{array}$ & Applications \\
\hline $1^{73}$ & PEDOT:PSS/xylitol & $50 \mathrm{wt} \%$ & $407 \mathrm{~S} \mathrm{~cm}^{-1}$ & $30 \%$ & - & \\
\hline $2^{74}$ & PEDOT:PSS/xyl & $50 \mathrm{wt} \%$ & $114 \mathrm{~S} \mathrm{~cm}^{-1}$ & $33 \%$ & - & \\
\hline $3^{76}$ & PEDOT:PSS/Zonyl & $90 \mathrm{wt} \%$ & $153 \Omega \mathrm{sq}^{-1}$ & $35 \%$ & $1.1-40 \%$ & $\begin{array}{l}\text { Organic solar cells } \\
\text { Wearable sensors }\end{array}$ \\
\hline $4^{77}$ & PEDOT:PSS/Triton X-100 & $99.3 \mathrm{wt} \%$ & $72 \mathrm{~S} \mathrm{~cm}^{-1}$ & $57 \%$ & $1.5-50 \%$ & Deformable electronics \\
\hline $5^{96}$ & PEDOT:PSS/D-Sorbitol & $97.7 \%$ & $>1000 \mathrm{~S} \mathrm{~cm}^{-1}$ & $63 \%$ & $2.2-60 \%$ & \\
\hline $6^{78}$ & $\begin{array}{l}\text { PEDOT:PSS/ionic } \\
\text { liquid (EMIM TCB) }\end{array}$ & 99 wt\% & $1000 \mathrm{~S} \mathrm{~cm}^{-1}$ & $28 \%$ & $\begin{array}{l}2-180 \% \text { (supported } \\
\text { on pre-strained PDMS) }\end{array}$ & Transparent electrodes \\
\hline $6^{79}$ & $\begin{array}{l}\text { PEDOT:PSS/ILs (EMIM DCI } \\
\text { and EMIM DCA) }\end{array}$ & & $538 \mathrm{~S} \mathrm{~cm}^{-1}$ & $23 \%$ & - & $\begin{array}{l}\text { Efficient organic } \\
\text { thermoelectrics } \\
\text { Wearable thermoelectrics }\end{array}$ \\
\hline $7^{80}$ & PEDOT:PSS/ILS BF4 & $38 \mathrm{wt} \%$ & $135 \mathrm{~S} \mathrm{~cm}^{-1}$ & & - & \\
\hline $8^{81}$ & $\begin{array}{l}\text { (PEDOT:PSS-PVP) fiber/IL } \\
\text { (EMIMAc) } \\
\text { Electrospun }\end{array}$ & $98.04 \mathrm{wt} \%$ & $1.8 \times 10^{-4} \mathrm{~S} \mathrm{~cm}^{-1}$ & $35 \%$ & $0.6-35 \%$ & \\
\hline $9^{82}$ & $\begin{array}{l}\text { PEDOT:PSS/PEO } \\
\text { PEG and PVA }\end{array}$ & $\begin{array}{l}33.3 \text { wt } \% \\
\text { (with PVA) } \\
33.3 \text { wt } \% \\
\text { (with PEO) }\end{array}$ & $\begin{array}{l}2 \times 10^{-4} \mathrm{~S} \mathrm{~cm}^{-1} \\
36 \mathrm{~S} \mathrm{~cm}^{-1}\end{array}$ & $\begin{array}{l}55 \% \\
36 \%\end{array}$ & $\begin{array}{l}- \\
-\end{array}$ & \\
\hline $10^{85}$ & $\begin{array}{l}\text { PEDOT:PSS/PVA } \\
\text { Blending }\end{array}$ & $50 \mathrm{wt} \%$ & $3 \mathrm{~S} \mathrm{~cm}^{-1}$ & $56.2 \%$ & - & \\
\hline $11^{86}$ & $\begin{array}{l}\text { PEDOT:PSS/PEO or } \\
\text { PVA blending }\end{array}$ & $60 \mathrm{wt} \%$ & $8.6 \times 10^{-6} \mathrm{~S} \mathrm{~cm}^{-1}$ & & - & \\
\hline $12^{88}$ & $\begin{array}{l}\text { PEDOT:PSS/PEO20- } \\
\text { PPO70-PEO20 (P123) } \\
\text { Blending }\end{array}$ & $80 \mathrm{wt} \%$ & $1700 \mathrm{~S} \mathrm{~cm}^{-1}$ & $40 \%$ & $1.3-40 \%$ & High transparency (89\%) \\
\hline $13^{97}$ & PEDOT:PSS/WPU & 92.5 wt\% & - & $200 \%$ & $>10^{3}-200 \%$ & $\begin{array}{l}\text { High transparency }(85.5 \%) \\
\text { dielectric elastomer actuators }\end{array}$ \\
\hline $13^{89}$ & $\begin{array}{l}\text { PEDOT:PSS/WPU } \\
\text { Blending }\end{array}$ & $20 \mathrm{wt} \%$ & $77 \mathrm{~S} \mathrm{~cm}^{-1}$ & $32.5 \%$ & - & High EMI shielding \\
\hline
\end{tabular}

the inter-spherulitic region, resulting in the formation of more conductive pathways. But the crystallization of soft polymers is unfavorable for the elongation at break. In the research by Ouyang and co-workers, ${ }^{82}$ the mechanical and electrical properties of the blends of these three polymers with PEDOT:PSS were systematically investigated and similar results were reported. Three kinds of polymer with a range of molecular weights (PEG20k $\left(M_{\mathrm{n}}=20 \mathrm{k}\right)$, PEO100k $\left(M_{\mathrm{v}}=100 \mathrm{k}\right)$, PEO1000k $\left(M_{\mathrm{v}}=1000 \mathrm{k}\right)$ and PVA89k $\left.\left(M_{\mathrm{w}}=89 \mathrm{k}-98 \mathrm{k}\right)\right)$ were blended with PEDOT:PSS, respectively. Although all the soft polymers have flexible polymer chains, it was observed that the elongation at break of PEG20k, PEO100k and PEO1000k is lower than that of PVA89k due to the high crystallinity of PEG20k and PEO100k. Meanwhile, the tensile strength decreases and the elongation at break increases obviously compared with neat PEDOT:PSS. The plastic deformation could be attributed to the plastic properties of soft materials, and PVA89k could improve both tensile strength and elongation at break due to the hydrogen bonds among the PVA chains (Fig. 2(c)). Unfortunately, the elasticity of these composites is rather poor since most of the tensile strain are non-recoverable (Fig. 2d). Subsequently, DMSO and EG were added into polymer blend solutions to further increase the conductivity. It was found that conductivity can be enhanced from $0.2 \mathrm{~S} \mathrm{~cm}^{-1}$ up to $75 \mathrm{~S} \mathrm{~cm}^{-1}$ for a PVA98k/ PEDOT:PSS 66 wt\% film after secondary treatment, while the elongation at break was slightly decreased as demonstrated in Fig. 2e. Besides these polymers, a triblock copolymer, poly(ethylene glycol)-block-poly(propylene glycol)-block-poly(ethylene glycol) (PEO20-PPO70-PEO20, Pluronic ${ }^{\circledR}$ P123), ${ }^{88}$ waterborne polyurethane (WPU), ${ }^{89}$ polyethylene-block-poly(ethylene glycol) (PBP) ${ }^{90,91}$ were also introduced into PEDOT: PSS aqueous to prepare stretchable conducting materials. Because PEDOT is a crystalline polymer, the aggregate structure (crystallinity, orientation) of PEDOT:SS will also affect the conductivity and mechanical properties of the overall materials. ${ }^{24,92}$ For pure PEDOT:PSS fibers via wet-spinning followed by hotdrawing and EG treatment, a record electrical conductivity of $2804 \mathrm{~S} \mathrm{~cm}^{-1}$ and superior mechanical properties with a Young's modulus of up to $8.3 \mathrm{GPa}$, a tensile strength of $409 \mathrm{MPa}$ and a large elongation of $21 \%{ }^{93}$ were achieved. The formation of highly ordered self-assembled domains of PEDOT chains was observed along with PEDOT:PSS fibers after hotdrawing. Fibers based on polymer blends such as PEDOT:PSS/ $\mathrm{PVA}^{94,95}$ have also been developed by wet spinning, followed by suitable coagulation treatment and hot-drawing. Zhou et al. prepared curled PEDOT/PBP fibers in thermoplastic elastomer tubes using the "solution stretch-dry-flexion" process to obtain highly stretchable coaxial fiber conductors. ${ }^{90}$ The unique axial layered flexion conducting core makes the resistance of the fiber very stable, with conductivity changes of less than $4 \%$ under up to $680 \%$ strain. They illustrated that it is important to control both the composition and morphology of PEDOT:PSS based blends through processing to achieve good electrical and mechanical properties. 
The electrical conductivity and stretchability of various PEDOT: PSS polymer blends are briefly summarized in Table 2. PEDOT: PSS blends containing small molecular plasticizers or ionic liquids exhibited an average elongation of $30 \%$ and maintained a high value of electrical conductivity. But due to the instability of these materials during subsequent application, the number of research studies based on such a strategy has gradually decreased. On the other hand, PEDOT:PSS blends containing soft matter exhibits good stretchability. However, due to the plasticity of these insulating soft materials and rather poor mechanical properties of PEDOT:PSS, reversible tensile properties cannot be achieved for these materials. Meanwhile, since these insulating polymers are miscible with PEDOT:PSS, they could prevent the phase continuity of the conductive polymer, and even wrap the PEDOT colloidal particles, thus preventing the formation of the PEDOT conductive network. From this perspective, it might be a paradox to maintain high conductivity while improving tensile properties only relying on a blending approach with miscible polymers.

\subsection{Phase separated polymer blends}

The second major route to fabricate stretchable electronic devices based on PEDOT:PSS is constructing a continuous conducting pathway embedded in an elastomeric matrix based on immiscible polymer blends. Nevertheless, blending PEDOT: PSS with hydrophobic elastomers is more difficult compared with the rest due to the poor compatibility between them. For example, PDMS is the most commonly used elastomer substrate in electronic skin and flexible wearable devices. PDMS and PEDOT blends are usually prepared by some specific means in most cases. With the support of an amphiphilic PDMS- $b$-PEO block copolymer, Jin-Seo Noh blended PEDOT: PSS with PDMS. ${ }^{98}$ Such a block copolymer greatly improved the miscibility between PEDOT:PSS and PDMS, and has an important influence on the phase morphology of these blends as shown in Fig. 3a. PEDOT:PSS and PDMS are completely phase separated without the addition of PDMSO- $b$-PEO. With increasing PDMSO- $b$-PEO content, the two phases gradually began to mix and the phase area size of isolated PDMS gradually decreased. Ultimately, PDMS granules were connected with each other to form a continuous network at a PDMS- $b$-PEO content of $30 \%$, whereas the PEDOT:PSS phase also formed a network structure. Although macroscopic phase separation was observed in both liquid and solid states, optimal PEDOT:PSS/ $30 \%$ PDMS films could be stretched up to $75 \%$ while maintaining a conductivity of $0.3 \mathrm{~S} \mathrm{~cm}^{-1}$. Meanwhile, a non-ionic surfactant, $p$-t-octylphenol (Triton X-100), was used to improve the miscibility of the two. ${ }^{99}$ The hydrophilic and hydrophobic ends of Triton X-100 are respectively connected with PEDOT: PSS aqueous solution and PDMS, so that PEDOT:PSS is uniformly dispersed in PDMS without obvious phase separation. Moreover, Triton X-100 can further improve the electrical conductivity of PEDOT:PSS on the basis of a secondary doping mechanism. As a result, the blend film exhibits an excellent piezoresistive effect and stability. The sheet resistance is $20 \Omega \mathrm{sq}^{-1}$ and the elongation is $82 \%$ for the composite film with $60 \%$ PDMS and 10\% Triton X-100. Apart from introducing a mediator to the mixing process, embedding PDMS oligomers and curing agents into a 3D PEDOT:PSS aerogel is also an effective strategy (Fig. 3b and c). The SEM images revealed that the blend film has an interpenetrating polymer network that comprise two types of 3D networks: a PEDOT:PSS aerogel network and a flexible PDMS network, which greatly improves the mechanical properties of PEDOT:PSS composites. The as-prepared PEDOT:PSS (aerogel)/PDMS conductors ${ }^{100}$ remain elastic under strain stress (43\%) and compressive stress (60\%), and it retains its electrical conductivity under deformation. The sheet resistance remained nearly constant $\left(2.5 \Omega \mathrm{sq}^{-1}\right)$ after 15 cycles of strain from $0 \%$ to $10 \%$ and 5000 cycles of folding.

As another type of polymer could be incorporated into PEDOT:PSS, cellulose nanofibers attract much attention on account of their dissolving system and abundant functional groups on the surface. They are mixed into PEDOT:PSS to facilitate the formation of a special polymer network with both good electrical and mechanical properties. For instance, flexible PEDOT:PSS/cellulose conductive electrodes ${ }^{101}$ could be constructed by sequential vacuum-assisted filtration of a solution containing these materials. From FE-SEM and AFM images, the composite film shows an inter-connected nanofiber morphology with PEDOT:PSS filled between the gaps. The electrical conductivity and the specific capacitance are $2.58 \mathrm{~S} \mathrm{~cm}^{-1}$ and $6.21 \mathrm{~F} \mathrm{~g}^{-1}$, respectively. CNF-PEDOT:PSS conducting nanopapers, ${ }^{102}$ PEDOT:PSS/bacterial-cellulose composite films, ${ }^{103}$ CNC-PEDOT:PSS blends, ${ }^{104}$ PEDOT:PSS/tempo-CNF films and aerogels ${ }^{105}$ have also been studied. For instance, Youngsan and co-workers ${ }^{78}$ prepared a CNF-PEDOT:PSS electrode using a vacuum assisted filtration method. As displayed in Fig. 4(a), the mixture suspension of PEDOT:PSS and CNF solution was filtrated on a PTFE filter, followed by drying. From the SEM images of the CNF-PEDOT:PSS film, it was observed that PEDOT:PSS is filled between CNF fibers to form conductive pathways (Fig. 4b) and the sheet resistance of the as-received film is $370 \mathrm{Ohm} \mathrm{sq}^{-1}$. Meanwhile, according to Zhou and co-workers, ${ }^{105}$ the electrical conductivity of the PEDOT:PSS/ CNF aerogel $\left(0.12 \mathrm{~S} \mathrm{~m}^{-1}\right.$ with $50 \%$ CNF loading) is higher than the neat PEDOT:PSS aerogel $\left(0.05 \mathrm{~S} \mathrm{~m}^{-1}\right)$ because the hydrogen bond between $-\mathrm{OH}$ of cellulose and $-\mathrm{SO}_{3}$ of PSS promotes the conformational transformation of PEDOT. They further infused a PEDOT:PSS/CNF aerogel with elastomer PDMS to form a strain sensor on the basis of the disconnection of conductive networks in the PEDOT:PSS/CNF aerogel under strain. The PDMS-infused conductive PEDOT:PSS/CNF aerogel (70 wt\% $\mathrm{CNF}$ ) is a stretchable, sensitive, and linearly responsive strain sensor, and the gauge factor reaches 14.8 at 95\% strain. Nevertheless, flexible PEDOT:PSS/cellulose films commonly suffer from limited conductivity. Rubber latex is also a candidate to blend with PEDOT:PSS, as both exist in water dispersion systems. Tai et al. firstly blended a PEDOT:PSS conductive dispersion with hydrophobic and rubbery poly(n-butyl acrylatestyrene) (P(BA-r-St)) latex-like elastomers. ${ }^{106}$ The as-prepared film illustrates an elongation of $97 \%$ and conductivity of $63 \mathrm{~S} \mathrm{~cm}^{-1}$ and transmittance of $93 \%$. But the mechanism for such high 

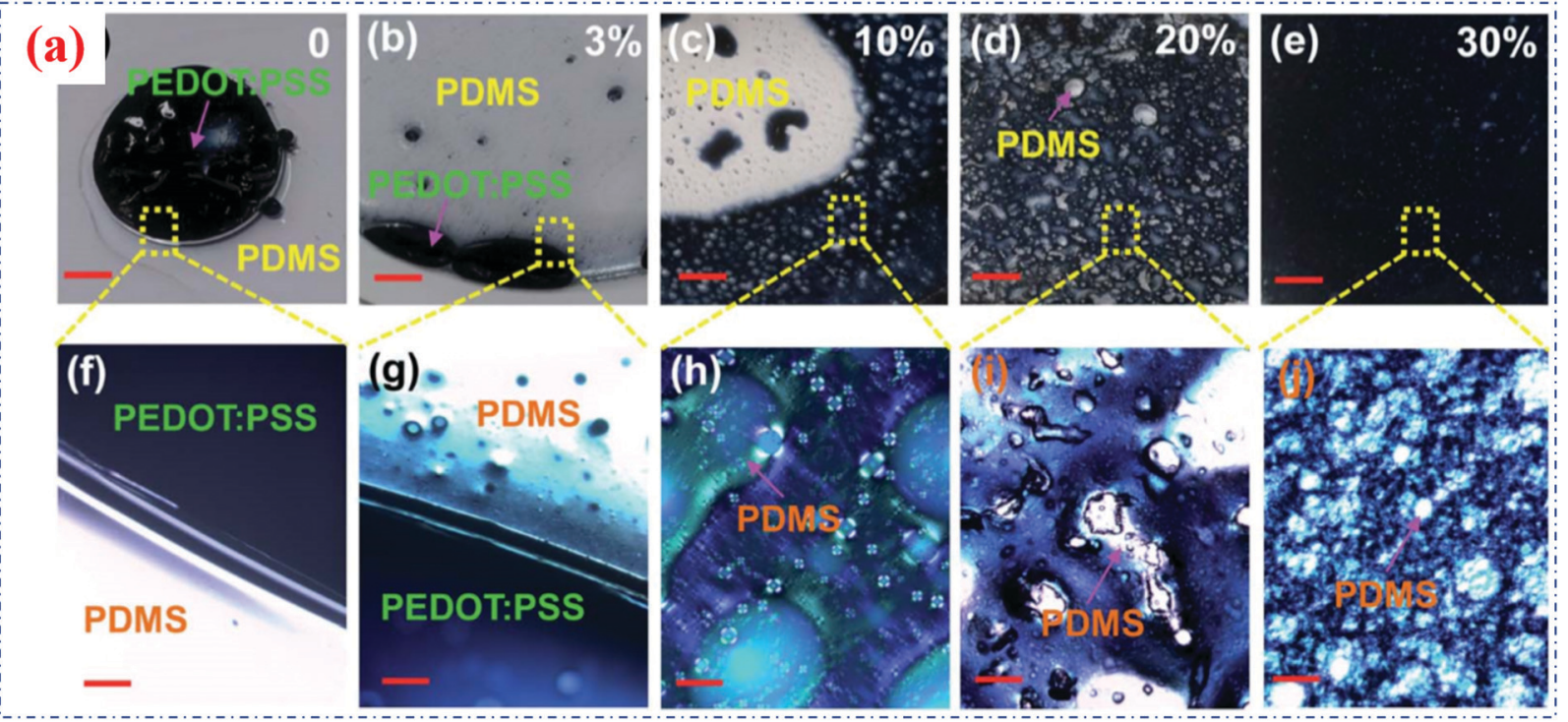

(b)

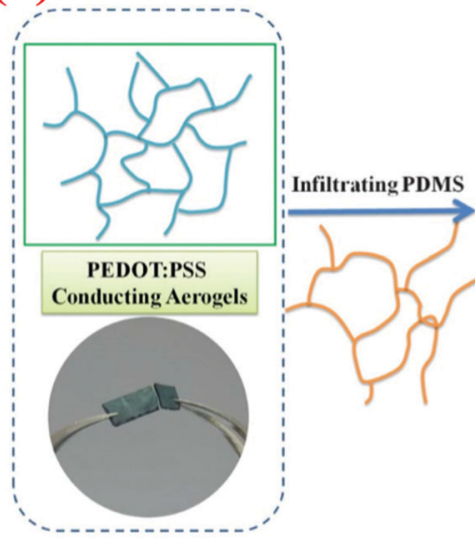

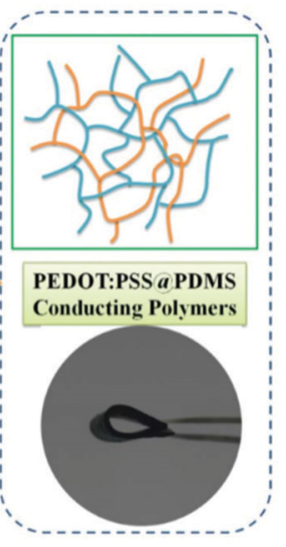

(c)

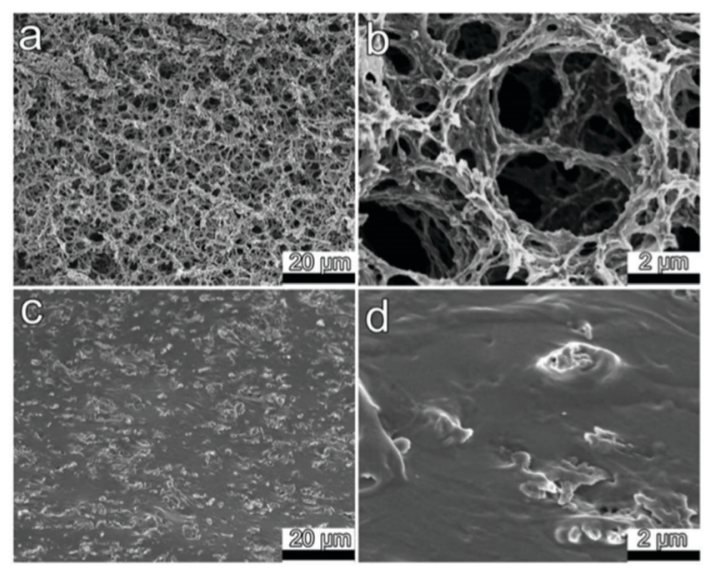

Fig. 3 (a) (a-e) Macroscopic images of PEDOT:PSS/PDMS mixtures with different concentrations of PDMS- $b$-PEO (0 to 30\%). The ratio of PEDOT:PSS to PDMS was $2: 1$ by weight for all samples. (f-j) Microscopic optical images of the selected areas of the corresponding macroscopic images ((a-e)). Scale bars are $5 \mathrm{~mm}$ for $(\mathrm{a}-\mathrm{e})$ and $30 \mathrm{~mm}$ for $(\mathrm{f}-\mathrm{j})$; (b) schematic showing the fabrication of PEDOT:PSS@PDMS conducting polymers; (c) SEM images of the PEDOT:PSS aerogel and PEDOT:PSS-PDMS film at different magnifications. Produced with permission. ${ }^{98,100}$ Copyright (C) 2013, Royal Society of Chemistry.

electrical conductivity and stretchability is unclear. On the other hand, Wang et al. ${ }^{107}$ used a carboxylated styrene-butadiene (XSB) latex blend with PEDOT:PSS for multi-functional touchless sensing. The blends were found to be composed of 3D XSB elastic networks and the PEDOT:PSS phase filled in between the networks (Fig. 4c-e). The composite with such a structure containing 1.6 wt\% PEDOT:PSS exhibits a high stretchability of up to $340 \%$ but ultralow conductivity $\left(<10^{-4} \mathrm{~S} \mathrm{~cm}^{-1}\right)$. It is worth noting that these composites show exceptional sensitivity toward different touchless stimuli such as humidity, temperature, and even NIR light. Taroni et al. ${ }^{108}$ blended commercial elastomeric polyurethane (Lycra) with freeze dried PEDOT:PSS in DMSO. The phase structure morphology of PEDOT:PSS and Lycra blends with different proportions was carefully characterized, where Lycra was dispersed as fibres with a diameter of around 100 microns in the PEDOT:PSS matrix. For a $10 \mathrm{wt} \%$ PEDOT:PSS-Lycra film, an unprecedented strain at break of $700 \%$ has been achieved.
Meanwhile, a high electrical conductivity of $79 \mathrm{~S} \mathrm{~cm}^{-1}$ and a Seebeck coefficient of $16 \mu \mathrm{V} \mathrm{K}^{-1}$ after EG post treatment were achieved (Fig. 4f-h). By stretching the film, the resistance increases, and then decreases during relaxation. Hence the blend is used to fabricate a soft strain sensor for detecting target motion. Furthermore, self-powered sensing is realized by utilizing the thermoelectric effect of PEDOT:PSS, where the composite films are able to detect tensile stress without any power supply.

Table 3 summarizes the properties of phase separated polymer blends discussed above. The average elongation at break can exceed $100 \%$ while maintaining a relatively high conductivity of $70 \mathrm{~S} \mathrm{~cm}^{-1}$. Besides, these phase separated polymer blends have versatile properties and functional characteristics, and they could be utilized in various components of flexible or stretchable electronics. Particularly for PEDOT: PSS and elastomer blends, they tend to exhibit good tensile 


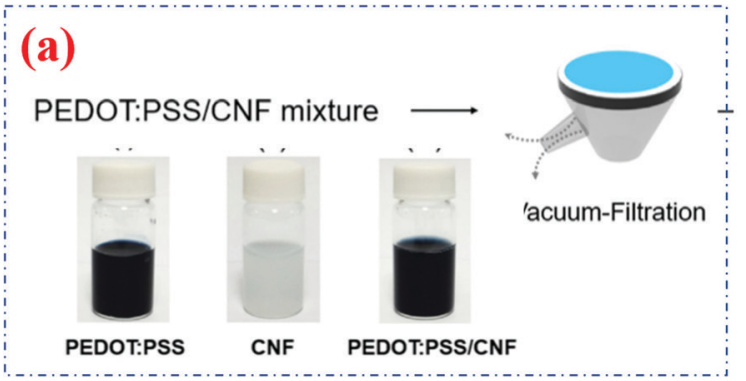

(c)

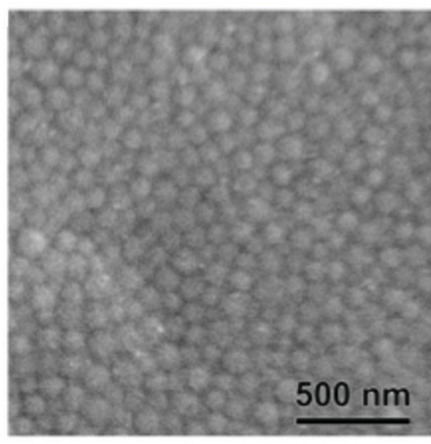

(d)

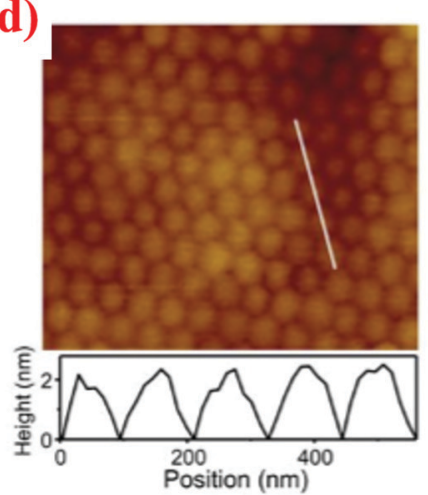

(f)

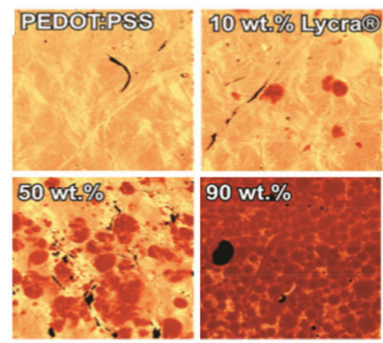

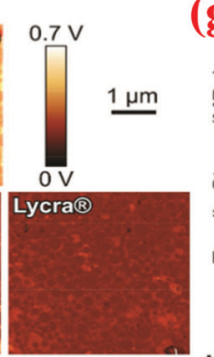

(b)
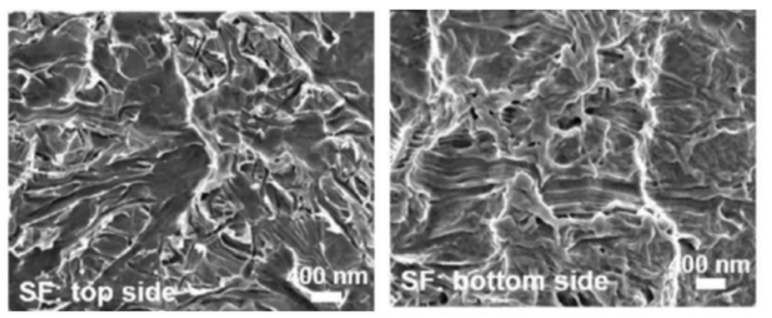

(e)

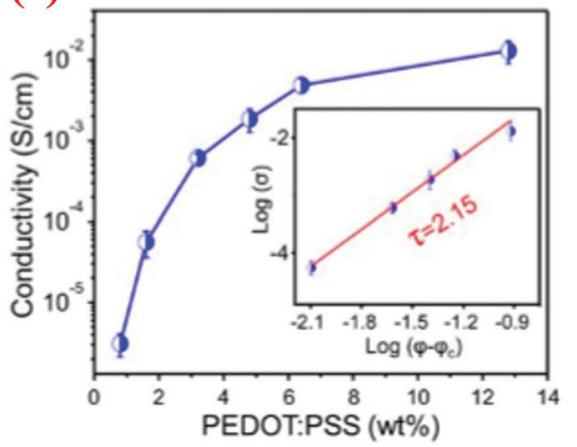

(h)

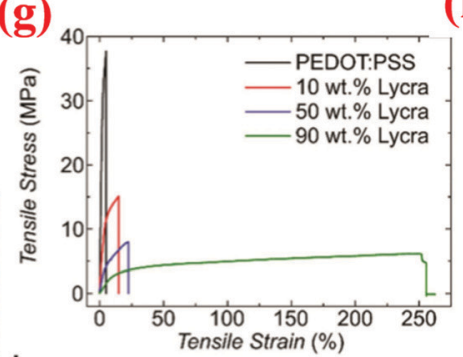

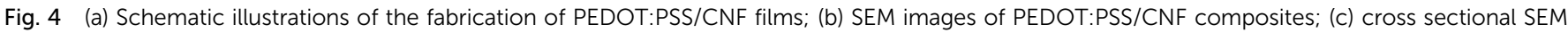

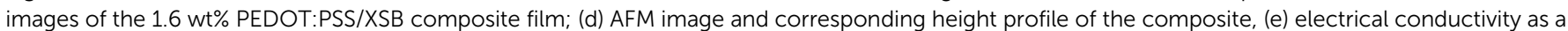

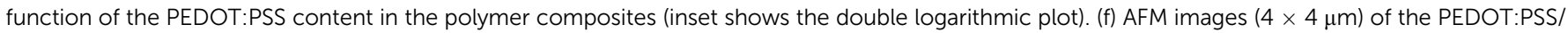

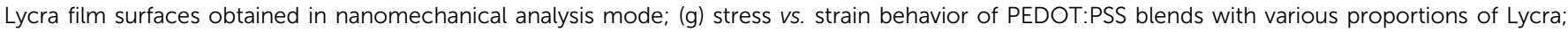

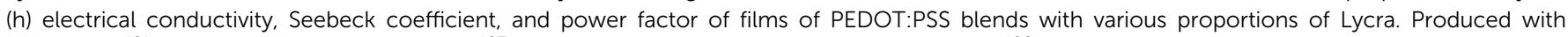

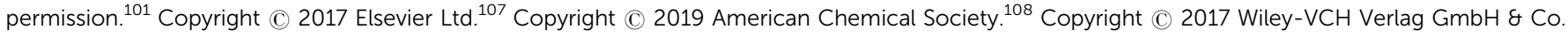
KGaA, Weinheim.

properties and partial tensile recovery. Therefore, such materials are often used as elastic conductors or strain sensitive sensors.

\section{PEDOT:PSS based polymer composites for thermoelectric application}

In order to meet the requirements under different application conditions, PEDOT:PSS could also be modified with various functional fillers. For example, although the conductivity of PEDOT:PSS can reach more than $1000 \mathrm{~S} \mathrm{~cm}^{-1}$ after secondary doping, there is still a large gap in the electrical conductivity compared with carbon nanotubes, graphene and other flexible materials. Besides, as an organic thermoelectric material (TE), PEDOT:PSS outperforms traditional inorganic counterparts because of its flexibility, light weight and ultralow thermal conductivity (usually in the range of $0.1-0.5 \mathrm{~W} \mathrm{~m}^{-1} \mathrm{~K}^{-1}$ ). ${ }^{111-113}$ But the thermoelectric properties of neat PEDOT:PSS are not comparable to those of inorganic semiconductor materials. The figure of merit (ZT) of PEDOT:PSS is 2-3 orders of magnitude lower than that of an inorganic semiconductor. Herein, the $Z T$ value is used to evaluate the efficiency of thermoelectric materials. It is a dimensionless quantity, which is defined as $Z T=$ $S^{2} \sigma T / \kappa$, where $S$ is the Seebeck coefficient, $\sigma$ is the electrical conductivity, $T$ is the absolute temperature, $\kappa$ is the thermal conductivity and $S^{2} \sigma$ represents the power factor (PF). In order to obtain a high $Z T$ value, materials require high conductivity, high thermoelectric coefficient and low thermal conductivity simultaneously. Indeed, there have been a number of literature reviews on strategies to improve the thermoelectric properties of PEDOT:PSS; ${ }^{114,115}$ for instance, de-doping, secondary doping, 
Table 3 Brief properties of current PEDOT:PSS blends and composites

\begin{tabular}{|c|c|c|c|c|c|c|c|}
\hline No. & Preparation & $\begin{array}{l}\text { PEDOT:PSS } \\
\text { content }\end{array}$ & $\begin{array}{l}\text { Conductivity or } \\
\text { sheet resistance }\end{array}$ & Elongation & $\begin{array}{l}\text { Resistance } \\
\text { change } \\
\left(R / R_{0} \text {-strain }\right)^{a}\end{array}$ & Applications & Other properties \\
\hline $1^{98}$ & $\begin{array}{l}\text { Blending (with PDMS- } g \text { - } \\
\text { PEO) }\end{array}$ & $30 \%$ & $0.3 \mathrm{~S} \mathrm{~cm}^{-1}$ & $75 \%$ & $2-75 \%$ & $\begin{array}{l}\text { Stretchable organic } \\
\text { interconnects }\end{array}$ & \\
\hline $2^{99}$ & $\begin{array}{l}\text { Blending (with EG/Triton } \\
\mathrm{X}-100 \text { ) }\end{array}$ & $60 \%$ & $80 \Omega \mathrm{sq}^{-1}$ & $82 \%$ & $1.5-75 \%$ & Stretchable LED circuits & $\begin{array}{l}\text { Piezoresistive effects } \\
\text { Long-term stability }\end{array}$ \\
\hline $3^{100}$ & $\begin{array}{l}\text { PEDOT:PSS } \\
\text { aerogel/PDMS }\end{array}$ & $7.1 \%$ & $\begin{array}{l}2.5 \Omega \mathrm{sq}^{-1} \\
(0-10 \% \text { cycle })\end{array}$ & $43 \%$ & $1.04-10 \%$ & $\begin{array}{l}\text { Flexible stretchable } \\
\text { displays }\end{array}$ & $\begin{array}{l}\text { Water stable electrical } \\
\text { properties }\left(2.1 \Omega \mathrm{sq}^{-1}\right)\end{array}$ \\
\hline $4^{102}$ & CNF-PEDOT:PSS & $38 \%$ & $10.55 \mathrm{~S} \mathrm{~cm}^{-1}$ & $1.6 \%$ & - & Energy storage devices & \\
\hline $5^{101}$ & $\begin{array}{l}\text { Simple vacuum-assisted } \\
\text { filtration } \\
\text { PEDOT:PSS-CNFs bilayer }\end{array}$ & $50 \%$ & $\begin{array}{l}22.6 \mathrm{~S} \mathrm{~cm}^{-1} \\
\text { (bilayer) }\end{array}$ & - & $\begin{array}{l}\text { 1-Bending } \\
100 \text { times }\end{array}$ & Conductive films & $\begin{array}{l}\text { Flexibility } \\
\text { Stability }\end{array}$ \\
\hline $6^{103}$ & $\begin{array}{l}\text { Blending PEDOT:PSS/ } \\
\text { bacterial cellulose }\end{array}$ & $31.24 \%$ & $12.17 \mathrm{~S} \mathrm{~cm}^{-1}$ & - & - & $\begin{array}{l}\text { Biosensors } \\
\text { OLED } \\
\text { Solar cells }\end{array}$ & \\
\hline $7^{104}$ & CNCs-PEDOT:PSS & $50 \%$ & $38 \mathrm{~S} \mathrm{~m}^{-1}$ & - & - & $\begin{array}{l}\text { Organic light emitting } \\
\text { diodes } \\
\text { Organic solar cells }\end{array}$ & \\
\hline $8^{105}$ & $\begin{array}{l}\text { (CNF-PEDOT:PSS) } \\
\text { aerogel/PDMS }\end{array}$ & $50 \%$ & $105 \mathrm{~S} \mathrm{~m}^{-1}$ & & $\mathrm{GF}=14.8$ & Strain sensor & \\
\hline $9^{106}$ & $\begin{array}{l}\text { PEDOT:PSS/P(BA-St): } \\
\text { blending }\end{array}$ & $17 \%$ & $63 \mathrm{~S} \mathrm{~cm}^{-1}$ & $97 \%$ & $\begin{array}{l}\text { 1.15-Bending } \\
100 \text { times }\end{array}$ & - & $\begin{array}{l}\text { Superior transmit- } \\
\text { tance }(93 \%)\end{array}$ \\
\hline & $\begin{array}{l}\text { PEDOT:PSS-PBA: } \\
\text { polymerization }\end{array}$ & $87 \%$ & $300 \mathrm{~S} \mathrm{~cm}^{-1}$ & - & $3-80 \%$ & - & $\begin{array}{l}\text { Transmittance } \\
(>80 \%)\end{array}$ \\
\hline $10^{107}$ & PEDOT:PSS/(XSB) Latex & $1.6 \mathrm{wt} \%$ & $<10^{-4} \mathrm{~S} \mathrm{~cm}^{-1}$ & $340 \%$ & - & $\begin{array}{l}\text { Multifunctional touch } \\
\text { sensing }\end{array}$ & Humidity \\
\hline & & & & & & Capacitors & $\begin{array}{l}\text { Temperature light } \\
\text { sensitivity }\end{array}$ \\
\hline $11^{108}$ & $\begin{array}{l}\text { Blending (PEDOT:PSS/ } \\
\text { Lycra) }\end{array}$ & $10 \mathrm{wt} \%$ & $60 \mathrm{~S} \mathrm{~cm}^{-1}$ & $260 \%$ & $1.6-50 \%$ & $\begin{array}{l}\text { Self-powered strain } \\
\text { sensing }\end{array}$ & $\begin{array}{l}\text { Thermoelectric } \\
\text { property }\end{array}$ \\
\hline $12^{109}$ & $\begin{array}{l}\text { Synthesis PSS- } b \text { - } \\
\text { PPEGMEA by RAFT } \\
\text { polymerization }\end{array}$ & - & $1.1 \mathrm{~S} \mathrm{~cm}^{-1}$ & $128 \%$ & - & $\begin{array}{l}\text { Stretchable and bio- } \\
\text { compatible; conductive } \\
\text { material }\end{array}$ & Water-enabled healing \\
\hline $13^{110}$ & $\begin{array}{l}\text { Fiber wet-spinning tech- } \\
\text { niques (PU/PEOT:PSS) }\end{array}$ & $13 \mathrm{wt} \%$ & $9 \mathrm{~S} \mathrm{~cm}^{-1}$ & $350 \%$ & $5-50 \%$ & $\begin{array}{l}\text { Strain sensing } \\
\text { Body movement } \\
\text { Monitoring }\end{array}$ & Elastic recovery \\
\hline
\end{tabular}

incorporating other TE additives and so on. However, the conflicting combination of these three parameters has some limitations on the optimization of $Z T$.

In existing studies, most of the PEDOT:PSS composites containing fillers are intended to be applied to flexible electronic devices with improved conductivity or thermoelectric performance (including improved conductivity and higher thermoelectric coefficients). There are two types of typical functional fillers: inorganic semiconductors with a high Seebeck coefficient and carbon fillers with high conductivity. The following part will take representative research works as examples to discuss the composition, structure, processing methods and corresponding properties of these two kinds of composites.

\subsection{Inorganic fillers}

To improve the thermoelectric properties, inorganic fillers with a high Seebeck coefficient including $\mathrm{Bi}_{2} \mathrm{Te}_{3}$-based alloys, tellurium (Te), oxides and silicon compounds are added to the PEDOT:PSS system. In general, to prepare bulk PEDOT:PSS composites, solution blending is used, and the mixture is then deposited on a flexible substrate or rigid surface by spraying, drop casting and rotary coating. Currently, the best commercially available inorganic TE materials are $\mathrm{Bi}_{2} \mathrm{Te}_{3}$ alloys, which exhibits an appealing electrical conductivity of $\sim 1000 \mathrm{~S} \mathrm{~cm}^{-1}$, Seebeck coefficient of $100-200 \mu \mathrm{V} \mathrm{K} \mathrm{K}^{-1}$ and a figure of merit $Z T$ close to $1 .^{116}$ Zhang et al. ${ }^{117}$ prepared $\mathrm{Bi}_{2} \mathrm{Te}_{3} /$ PEDOT:PSS composites; ball milled $\mathrm{Bi}_{2} \mathrm{Te}_{3}$ powders were dispersed in alcohol and drop-cast on a glass substrate. Then, PEDOT:PSS aqueous was drop-cast on the $\mathrm{Bi}_{2} \mathrm{Te}_{3}$ thin film. The two-step drop casting method results in composites consisting of two layers: an upper layer of PEDOT:PSS and a lower layer of $\mathrm{Bi}_{2} \mathrm{Te}_{3}$ (Fig. 5a), leading to a power factor of around $47 \mu \mathrm{W} \mathrm{m} \mathrm{m}^{-1} \mathrm{~K}^{-2}$. Song et al. ${ }^{118}$ prepared $\mathrm{Bi}_{2} \mathrm{Te}_{3} /$ PEDOT:PSS composites by mixing $\mathrm{Bi}_{2} \mathrm{Te}_{3}$ powders with PEDOT:PSS aqueous directly. The effect of $\mathrm{Bi}_{2} \mathrm{Te}_{3}$ loading on the thermoelectric properties of composites was systematically investigated. There is an optimal value for $\mathrm{Bi}_{2} \mathrm{Te}_{3}$ loading in terms of the conductivity of composite materials. The conductivity first increases with increasing $\mathrm{Bi}_{2} \mathrm{Te}_{3}$ loading and reaches the maximum $\left(421 \mathrm{~S} \mathrm{~cm}^{-1}\right)$ at a weight fraction of $10 \mathrm{wt} \%$ and then decreases with further increase in the loading of $\mathrm{Bi}_{2} \mathrm{Te}_{3}$ powders. The addition of $\mathrm{Bi}_{2} \mathrm{Te}_{3}$ might provide a carrier to attach PEDOT:PSS, and the conformation of PEDOT chains is changed from coiled to linear, which possesses a higher electrical conductivity. Unfortunately, the mechanism is not clearly identified in this work. Excessive filler would lead to aggregation, making charge carrier transport more difficult. 

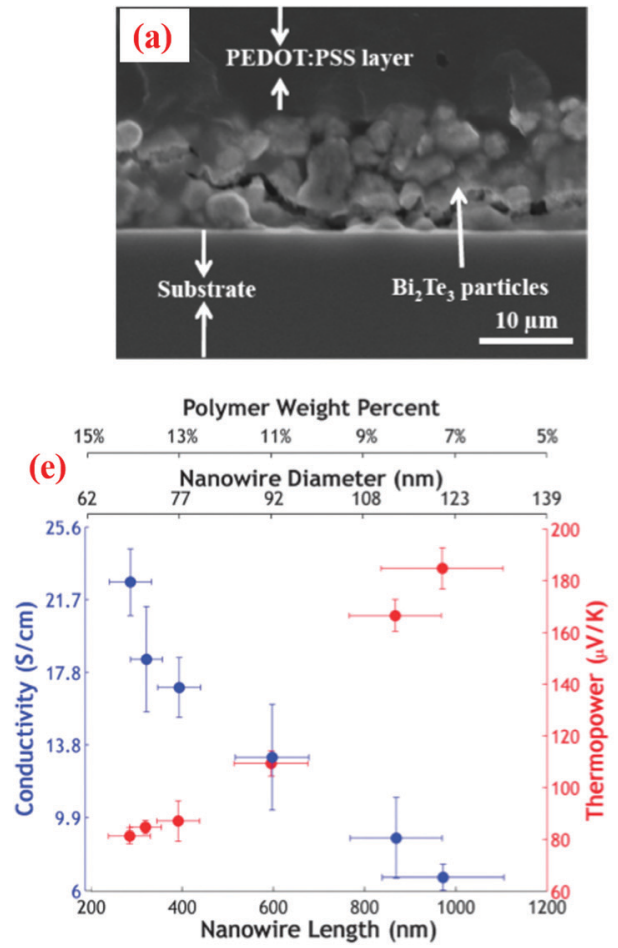
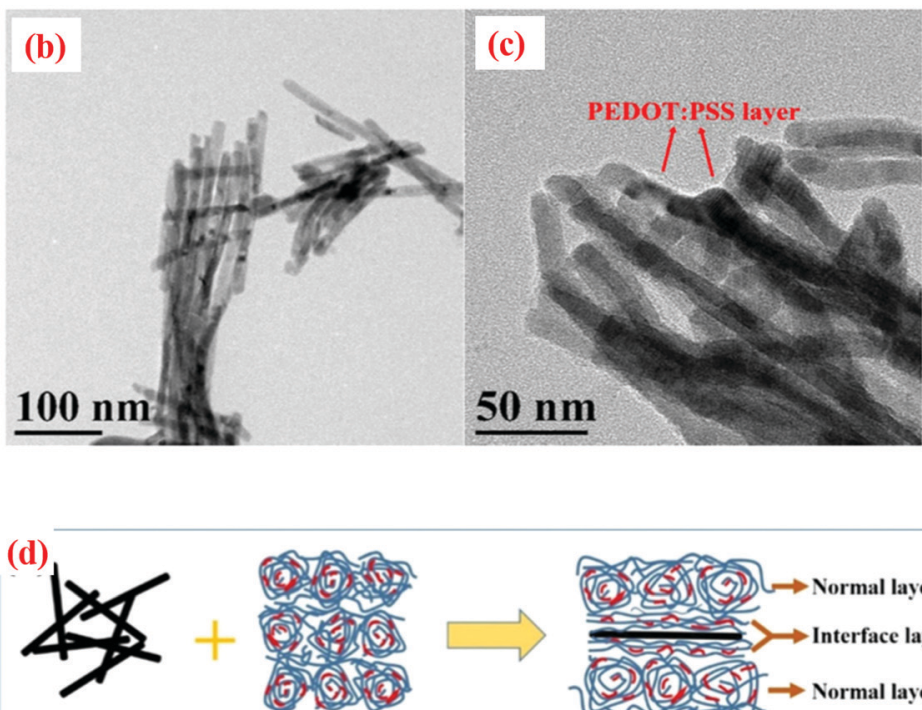

$\mathrm{Te}$

nanorods
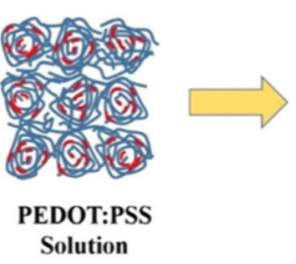

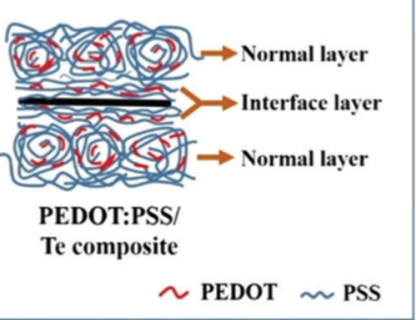

Fig. 5 (a) SEM images of cross section of $\mathrm{Bi}_{2} \mathrm{Te}_{3}$ /PEDOT:PSS composites; AFM images of (b) Te nanorods and (c) Te nanorod hybrid with PEDOT:PSS; (d) a schematic illustration of the preparation procedure of PEDOT:PSS/Te composite films; (e) nanowire length dependent properties of Te nanowire/ PEDOT:PSS composites. Produced with permission. ${ }^{117}$ Copyright (C) 2010 American Chemical Society. ${ }^{125}$ Copyright (C) 2016, Springer Nature. ${ }^{124}$ Copyright (C) 2013, Royal Society of Chemistry.

The $\mathrm{Bi}_{2} \mathrm{Te}_{3}$ loading had little effect on the Seebeck coefficient. Because PEDOT:PSS is still a continuous phase in the composite, the Seebeck coefficient of the composite is comparable to that of pure PEDOT:PSS $\left(\sim 15 \mu \mathrm{V} \mathrm{K} \mathrm{K}^{-1}\right)$. Subsequently, PEDOT:PSS composites with other inorganic particles such as $\mathrm{Bi}_{0.5} \mathrm{Sb}_{1.5} \mathrm{Te}_{3}$ particles, ${ }^{119} \mathrm{Ca}_{3} \mathrm{Co}_{4} \mathrm{O}_{9}$ nanoparticles, ${ }^{120} \mathrm{ZnO}_{2}$ flowers, ${ }^{121}$ and $\mathrm{Fe}_{3} \mathrm{O}_{4}$ nanoparticles ${ }^{122}$ have also been explored. Dispersing these fillers in the PEDOT:PSS matrix is a challenge because physicochemical properties of such organic/inorganic components are quite different. The agglomeration of fillers would reduce the amount of inorganic conductive network. In addition, the contact resistance on the surface of organic and inorganic materials will greatly limit further improvement in the conductivity of the overall composites. To address such issues, Shin and co-workers ${ }^{123}$ introduced a surfactant, Triton X-100 (TX100), to disperse $\mathrm{Bi}_{0.5} \mathrm{Sb}_{1.5} \mathrm{Te}_{3}$ in PEDOT:PSS homogeneously in an auxiliary manner. Triton X-100 improves the charge carrier transport through a more homogeneous dispersion and leads to efficient interfacial charge transport between $\mathrm{Bi}_{0.5} \mathrm{Sb}_{1.5} \mathrm{Te}_{3}$ and PEDOT. Simultaneously increased Seebeck coefficient and electrical conductivity are obtained, resulting in a maximum power factor of $40 \mathrm{~mW} \mathrm{~m} \mathrm{~m}^{-1} \mathrm{~K}^{-2}$. This study indicates the importance of interfacial engineering on physical mixed inorganic filler/ PEDOT:PSS composites for thermoelectric applications. Nevertheless, the effect of filler surface modification on the thermoelectric properties for PEDOT:PSS based composites is still unclear, and needs to be further studied.
In addition to $0 \mathrm{D}$ particles, 1D nanorods/nanowire/fillers have also been incorporated into PEDOT:PSS to achieve composites with high thermoelectric properties. Te exhibits a high Seebeck coefficient of $408 \mu \mathrm{V} \mathrm{K}^{-1}$ at room temperature, and it can be easily synthesized by thermal reduction. Segalman and co-workers ${ }^{124}$ synthesized Te nanorods in the presence of PEDOT:PSS. The composite films demonstrate an electrical conductivity of $19.3 \mathrm{~S} \mathrm{~cm}^{-1}$ and a Seebeck coefficient of $163 \mu \mathrm{V} \mathrm{K}^{-1}$ while retaining a polymeric thermal conductivity. The resulting power factor could be as high as $70 \mu \mathrm{W} \mathrm{m} \mathrm{m}^{-1} \mathrm{~K}^{-2}$. Nevertheless, the mechanism responsible for the above thermoelectric properties is still unclear. To further explore the mechanism, Song et al. ${ }^{125}$ coated a thin layer of PEDOT/PSS (about $3 \mathrm{~nm}$ ) on the surface of Te nanorods, and the PEDOT: PSS layer was in a highly ordered state and was templated by Te nanorods (Fig. 5(b-d)). The highly oriented PEDOT chains may promote carrier transport in the composite. In order to further optimize the thermoelectric properties of $\mathrm{Te}$ nanowire/ PEDOT:PSS composites, Segalman's group ${ }^{124}$ studied three influencing factors, namely, the morphology of Te nanowires, EG or DMSO post treatment of PEDOT:PSS matrix and the amount of polymer in composites. They found that EG or DMSO treatment can improve the electrical conductivity markedly without decreasing the Seebeck coefficient. A 16 wt\% PEDOT:PSS hybrid with long Te nanowires demonstrated a large power factor of $100 \mu \mathrm{W} \mathrm{m}{ }^{-1} \mathrm{~K}^{-2}$ (Fig. 5(e)). Besides the above issues, preparation methods would also influence the 
microstructure of the composite film, thus achieving different properties. For instance, Xiong and co-workers ${ }^{126}$ prepared $\mathrm{Bi}_{2} \mathrm{Te}_{3}$ nanowires-PEDOT:PSS composites with three structures: layer-by-layer (drop casting $\mathrm{Bi}_{2} \mathrm{Te}_{3}$ nanowires and PEDOT:PSS layer), hybrid (suspending $\mathrm{Bi}_{2} \mathrm{Te}_{3}$ nanowires in PEDOT:PSS aqueous) and pellet structures (tableting a $\mathrm{Bi}_{2} \mathrm{Te}_{3}$ pellet and drop casting PEDOT:PSS in it). The hybrid method achieved the highest electrical conductivity because it can obtain a compact film, where PEDOT:PSS and fillers are closely combined, and PEDOT:PSS can accommodate more filler loading under uniform dispersion conditions. The Seebeck coefficient of the layer-bylayer method $\left(14.9 \mu \mathrm{V} \mathrm{K}^{-1}\right)$ and hybrid method $\left(16.3 \mu \mathrm{V} \mathrm{K}{ }^{-1}\right)$ is close to that of neat PEDOT:PSS $\left(12.6 \mu \mathrm{V} \mathrm{K}^{-1}\right)$, indicating that the transport contributing to the Seebeck coefficient mostly depends on PEDOT:PSS, while the pellet method exhibits a higher Seebeck coefficient of $56.8 \mu \mathrm{V} \mathrm{K}^{-1}$. It is confirmed that PEDOT:PSS can enhance the carrier concentration and $\mathrm{Bi}_{2} \mathrm{Te}_{3}$ can increase the carrier mobility of the $\mathrm{Bi}_{2} \mathrm{Te}_{3}$ nanowires-PEDOT:PSS composites. The hybrid method achieved the highest power factor of $10.6 \mu \mathrm{W} \mathrm{m}{ }^{-1} \mathrm{~K}^{-2}$ because this method leads to a homogeneous structure for the composite film.

Moreover, 2D inorganic fillers are also promising candidates for PEDOT:PSS thermoelectric materials. Typical 2D materials like $\mathrm{MoS}_{2},{ }^{127,128} \mathrm{BN},{ }^{129} \mathrm{SnSe},{ }^{130} \mathrm{MoSe}_{2},{ }^{131} \mathrm{Bi}_{2} \mathrm{Te}_{3}$ nanosheets ${ }^{132}$ and black phosphorus ${ }^{133}$ have been introduced into the PEDOT: PSS matrix due to their high Seebeck coefficient and in-plane carrier mobilities at room temperature. $\mathrm{MoS}_{2} / \mathrm{PEDOT}$ :PSS, ${ }^{134}$ $\mathrm{MoSe}_{2}$ nanosheets/PEDOT:PSS composites ${ }^{131}$ thin films were prepared through mixing organic liquid-phase exfoliated $\mathrm{MoS}_{2}$ nanosheets with PEDOT:PSS solutions directly, then assisted by vacuum filtration (Fig. 6a). The organic solvent (DMSO, DMF) can partially remove PSS during the film-formation process and significantly enhance the electrical conductivity of PEDOT:PSS despite the negative effect of $\mathrm{MoS}_{2}$ and $\mathrm{MoSe}_{2}$ on conductivity. The Seebeck coefficient was largely enhanced due to the energy filtering effect between organic and inorganic materials. ${ }^{135-137}$ As a result, the maximum power factor was calculated to be $48.6 \mu \mathrm{W} \mathrm{m}^{-1} \mathrm{~K}^{-2}\left(\sigma \sim 1300 \mathrm{~S} \mathrm{~cm}^{-1}, S \sim 21 \mu \mathrm{V} \mathrm{K}{ }^{-1}\right)$ for $5 \mathrm{wt} \%$ $\mathrm{MOSe}_{2} / \mathrm{PEDOT}$ :PSS and $45.6 \mu \mathrm{W} \mathrm{m}^{-1} \mathrm{~K}^{-2}\left(\sigma \sim 1250 \mathrm{~S} \mathrm{~cm}^{-1}\right.$, $S \sim 19.5 \mu \mathrm{V} \mathrm{K}^{-1}$ ) for $4 \mathrm{wt} \% \mathrm{MoS}_{2} /$ PEDOT:PSS composites. On account of their simple process and excellent properties, $\mathrm{MOS}_{2} / \mathrm{PEDOT}$ :PSS composites are widely used in solar cells as efficient counter electrodes, ${ }^{138}$ humidity sensors, ${ }^{139}$ and supercapacitors. ${ }^{140}$ Meanwhile, Du et al. ${ }^{141}$ used spin coating and drop casting techniques to prepare $\mathrm{Bi}_{2} \mathrm{Te}_{3}$ nanosheets/ PEDOT:PSS composite films, respectively. The preparation technique and surface roughness of these films have much influence on the thermoelectric properties. Nanometer sized structures and tough surfaces of spin coated films make their electrical conductivity much lower than those of drop cast films. Drop cast composite films possess a high electrical conductivity of $1295 \mathrm{~S} \mathrm{~cm}^{-1}$ and a maximum power factor of $32.3 \mu \mathrm{W} \mathrm{m}^{-1} \mathrm{~K}^{-2}$. To widen their application, $\mathrm{Ou}$ and co-workers ${ }^{142}$ developed an aerosol jet printing method by printing bespoke inks consisting of PEDOT:PSS (Fig. 6(b)), $\mathrm{Bi}_{2} \mathrm{Te}_{3}$, and $\mathrm{Sb}_{2} \mathrm{Te}_{3}$ onto flexible polyimide substrates. With $85 \% \mathrm{Sb}_{2} \mathrm{Te}_{3}$ nanoflakes, the power
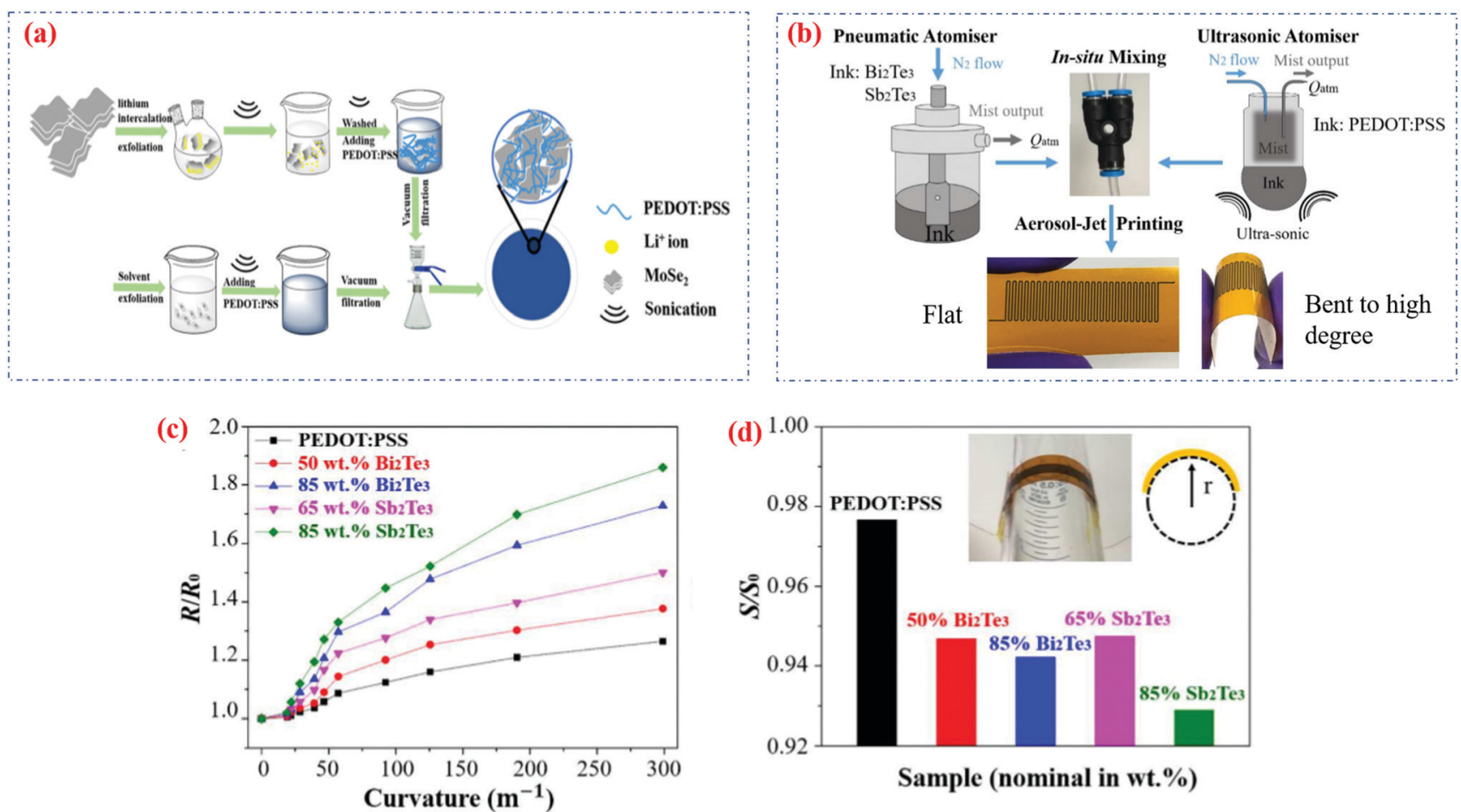

Fig. 6 (a) Schematic illustration of the preparation of the MoSe 2 /PEDOT:PSS composite; (b) schematic of the in situ mixing and aerosol jet printing method; ( $c$ and d) flexing test of printed $\mathrm{Bi}_{2} \mathrm{Te}_{3}$, and $\mathrm{Sb}_{2} \mathrm{Te}_{3} /$ PEDOT:PSS nanocomposites. Produced with permission. ${ }^{100}$ Copyright $\mathbb{C}$ ) 2017 IOP Publishing Ltd. ${ }^{156}$ Copyright (C) 2018 American Chemical Society. 
factor of the composite films reaches $28.3 \mu \mathrm{W} \mathrm{m}{ }^{-1} \mathrm{~K}^{-2}(\sigma \sim$ $247 \mathrm{~S} \mathrm{~cm}^{-1}, S \sim 33.8 \mu \mathrm{V} \mathrm{K}^{-1}$ ). Besides, variations in conductivity and Seebeck coefficient have been studied with varying degrees of bending and the composites demonstrated excellent mechanical properties as shown in Fig. 6(c and d), which gives these printed organic-inorganic nanocomposites a wider range of applications, particularly in wearable electronics. Besides, researchers also introduced a third-phase polymer to the composites to increase the flexibility. Zhang et al. $^{143}$ incorporated PVA to a PEDOT:PSS/ $\mathrm{Bi}_{0.5} \mathrm{Sb}_{1.5} \mathrm{Te}_{3}$ hybrid to improve the tensile strain. It was observed that the Seebeck coefficient drops to $24.1 \%$ for a strain of $25 \%$, which can be explained by a decrease in the number or quality of interfacial junctions between $\mathrm{Bi}_{0.5} \mathrm{Sb}_{1.5} \mathrm{Te}_{3}$ and PEDOT:PSS chains under strain.

The electrical conductivity of metal materials is as high as $10^{5} \mathrm{~S} \mathrm{~cm}^{-1}$, much higher than that of PEDOT:PSS. Thus, incorporating metal particles into the composites is an effective method to improve the conductivity of PEDOT:PSS. Au nanoparticles (spherical and rod shaped) ${ }^{144,145}$ and $\mathrm{Ag}$ nanowires $^{146,147}$ were mixed with PEDOT:PSS to obtain highly conductive composites. Toshima et al. ${ }^{148}$ prepared different shapes of Au particles and incorporated in a PEDOT:PSS system to obtain thermoelectric materials. The results show that the conductivity of nanorod-Au/PEDOT:PSS composites has been greatly improved $\left(\sim 2000 \mathrm{~S} \mathrm{~cm}^{-1}\right)$, which indicates that $1 \mathrm{D}$ particles with a larger aspect ratio are conducive to the preparation of conductive composites thanks to the percolation effect. Meanwhile, Liu et al. ${ }^{149}$ prepared high-performance Ag NWs/PEDOT:PSS thermoelectric composites through a spark plasma sintering process. Loading of $\mathrm{Ag}$ NWs increases the electrical conductivity ( $\sim 84 \mathrm{~S} \mathrm{~cm}^{-1}$ for $20 \mathrm{wt} \% \mathrm{Ag} \mathrm{NWs}$ ) without a noticeable decrease in the Seebeck coefficient. The $Z T$ value is 0.024 at room temperature. In addition to serving as a thermoelectric, Ag NWs/PEDOT:PSS composites as functional layers, coatings or components are widely applied in flexible organic solar cells, ${ }^{150}$ supercapacitors, ${ }^{151}$ electrodes, ${ }^{152-154}$ and flexible sensors. ${ }^{152,155}$ For instance, Yue et al. ${ }^{147}$ prepared transparent and conductive PEDOT:PSS/Ag NW/PEDOT:PSS three layer hybrid films by spin-coating. They exhibit a low sheet resistance of $21.9 \Omega \mathrm{sq}^{-1}$ and a high transmittance of $83.95 \%$ at $550 \mathrm{~nm}$ wavelength. Due to the excellent electrical conductivity of PEDOT and its wide application, Ag NWs/ PEDOT:PSS solution has become a commercially available product (Clevios).

In general, incorporating inorganic semiconducting fillers with high Seebeck coefficient and power factor into a PEDOT: PSS matrix is an efficient way to optimize the thermoelectric properties. We selected the most representative work and summarized the effects of different filler morphologies and preparation process on the thermoelectric properties of PEDOT:PSS composites. It could be concluded that the preparation method and the structure of composite films are the main factors that influence the thermoelectric properties of PEDOT:PSS based composites. A homogeneous dispersion of semiconductor fillers is beneficial for the improvement of Seebeck coefficient due to the energy filtering effect on the conjunction of the semiconductor and PEDOT:PSS matrix. And higher electrical conductivity can be acquired upon forming more conductive pathways. Compared with 0D powder fillers, 1D fillers with a high length-diameter ratio are more likely to improve conductivity because it can template PEDOT and PSS conformation from a coiled to a linear structure, thus a highly conductive PEDOT interlayer is formed at the interface between the inorganic filler and PEDOT:PSS matrix. Overall, understanding and controlling the interfacial interaction between the filler and matrix are extremely important for optimizing properties of organic/inorganic hybrid systems. Unfortunately very few research discussed these influencing factors ${ }^{135}$ for such type of composite. Most studies reported the preparation process and thermoelectric properties of these hybrid films, but the transport mechanism in organic/inorganic composites is unclear and research on it is in its infancy.

\subsection{Carbon fillers}

It is well known that carbon nanomaterials have excellent electrical properties. So carbon fillers such as carbon nanotubes (CNTs), ${ }^{157-161}$ graphene and carbon black fillers have been blended with PEDOT:PSS matrixes to optimize their electrical, thermal, mechanical, thermoelectric and other properties to meet their application requirements in different situations or to broaden their application range. CNTs have a stable 1D nanostructure and excellent electrical properties $\left(\sigma \sim 2 \times 10^{5} \mathrm{~S} \mathrm{~cm}^{-1}\right.$ at room temperature $\left.{ }^{162}\right)$. For PEDOT:PSS containing CNTs, due to the large specific surface area of CNTs and strong $\pi-\pi$ interactions between CNTs and PEDOT chains, effective interfacial contacts are formed between CNTs and PEDOT:PSS. They can be rather easily dispersed in PEDOT:PSS matrixes. ${ }^{163}$ As a result, their thermoelectric properties are affected. Yu et al. ${ }^{164}$ mixed single-walled carbon nanotubes (SWCNTs) with PEDOT:PSS and achieved an ultrahigh electrical conductivity of $\sim 10^{3} \mathrm{~S} \mathrm{~cm}^{-1}$ at room temperature, a stable Seebeck coefficient (27-41 $\left.\mu \mathrm{V} \mathrm{K}^{-1}\right)$ and an extremely high power factor of $160 \mu \mathrm{W} \mathrm{m}{ }^{-1} \mathrm{~K}^{-2}$ (Fig. 7(b)). The author suggested that the excellent thermoelectric properties can be attributed to the junction formed by SWCNTs and PEDOT:PSS. The junction can filter low energy electrons, thus maintaining the Seebeck coefficient. It also allows electrons to pass through the composite, increasing the conductivity. Meanwhile, it suppresses heat transport due to differences in vibrational spectra between CNTs and PEDOT:PSS, thus, the thermal conductivity is relatively low (Fig. 7a). Different from a rather homogeneous distribution, Jiang and co-workers ${ }^{165}$ prepared a SWCNTs/ PEDOT:PSS thin film through vacuum filtering PEDOT:PSS aqueous on a SWCNTs layer, obtaining a layered like structure. The power factor of a $60 \mathrm{wt} \%$ SWCNT/PEDOT:PSS hybrid film was $105 \mu \mathrm{W} \mathrm{m} \mathrm{K}^{-1} \mathrm{~K}^{-2}\left(\sigma \sim 600 \mathrm{~S} \mathrm{~cm}^{-1}, S \sim 42 \mu \mathrm{V} \mathrm{K} \mathrm{K}^{-1}\right)$. Subsequently, transparent electrodes ${ }^{160,166}$ and PEDOT:PSSCNT aerogels ${ }^{167}$ have been developed. It can be concluded that the transport feature of SWCNTs and PEDOT:PSS may lead to a power factor close to those of inorganic semiconductors such as bulk PbTe $(0.02 \text { at } 300 \mathrm{~K})^{168}$ or bulk silicon $(Z T \sim 0.01$ at $300 \mathrm{~K}){ }^{169}$ Meanwhile, graphene and reduced graphene oxide (rGO) are also 
(a)

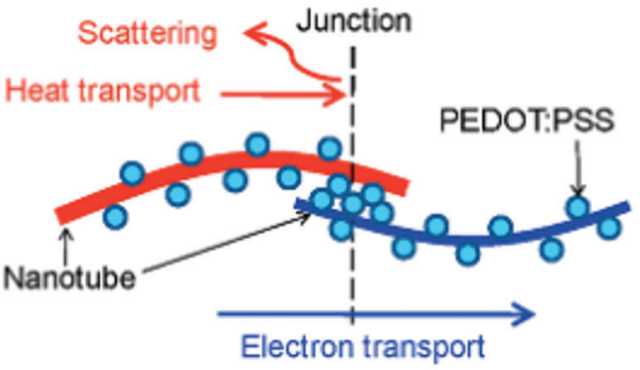

(b)
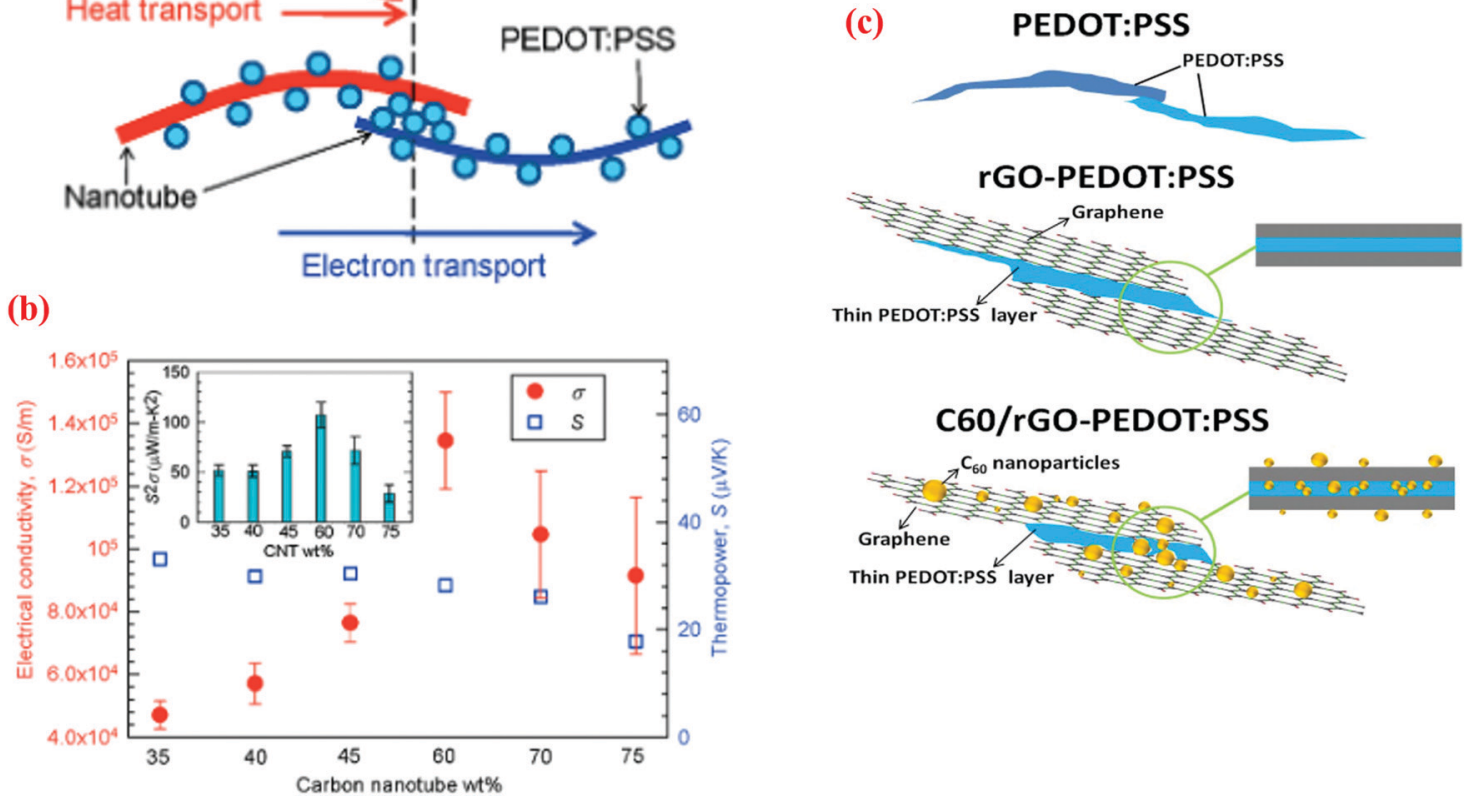

C60/rGO-PEDOT:PSS

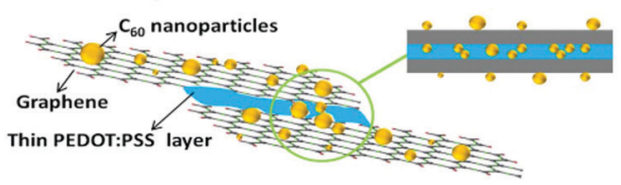

Fig. 7 (a) CNT-PEDOT:PSS-CNT junctions in the composites; (b) electrical conductivities and thermo-power of the composites with increasing CNT concentration. The inset shows the thermoelectric power factors $\left(S^{2} \sigma\right)$; (c) schematic illustration of carrier transport in the neat polymer film, rGOpolymer and $\mathrm{C}_{60} / \mathrm{rGO}$ nanohybrid-filled polymer composites. Produced with permission. Produced with permission. ${ }^{164}$ Copyright $\mathbb{C} 2011$ American Chemical Society. ${ }^{172}$ Copyright (C) 2013 Springer Nature.

among the most attractive fillers to prepare polymer-based conductors due to their appealing electrical conductivity. The preparation of graphene/PEDOT:PSS composites is often through ink preparation, and then inject printing, spraying or depositing onto a certain component such as a counter electrode in solar cells ${ }^{170}$ and organic photodetectors. Related studies have been well reviewed by Zhou et al..$^{35}$ The thermoelectric properties of rGO/PEDOT:PSS were investigated by Li et al. ${ }^{171}$ According to them, the maximum power factor is $\sim 32.6 \mu \mathrm{W} \mathrm{m}{ }^{-1} \mathrm{~K}^{-2}$ for a $3 \mathrm{wt} \%$ GO/PEDOT:PSS film after reduction. The electrical conductivity is as high as $1160 \mathrm{~S} \mathrm{~cm}^{-1}$ with $5 \% \mathrm{rGO}$. When the content of rGO increases, rGO flakes tend to aggregate, resulting in decreased electrical conductivity. The carrier transport mechanism was illustrated in Wang's work. ${ }^{172}$ They comparatively studied the electrical properties of $\mathrm{C}_{60} / \mathrm{rGO}-\mathrm{PEDOT}$ :PSS composites. It is observed that rGO is beneficial for improving the electrical conductivity while $\mathrm{C}_{60}$ (with ultrahigh $S \sim 2000 \mu \mathrm{V} \mathrm{K}^{-1}$ ) enhances the Seebeck coefficient and hinder the thermal conductivity of PEDOT:PSS. With the incorporation of a $\mathrm{C}_{60}-\mathrm{rGO}$ nano-hybrid, the Seebeck coefficient is enhanced by around 4 times and the electrical conductivity increases from $10000 \mathrm{~S} \mathrm{~m}^{-1}$ to $70000 \mathrm{~S} \mathrm{~m}^{-1}$ in comparison with that of neat PEDOT:PSS, resulting a high ZT value of $0.067 \mu \mathrm{W} \mathrm{m}^{-1} \mathrm{~K}^{-2}$. The mechanism of its carrier transport is demonstrated in Fig. 7 (c). The strong electron scattering of $\mathrm{C}_{60}$ particles assembled on graphene surfaces via $\pi-\pi$ stacking would compromise the electron mobility, thus reducing the electrical conductivity. However, it increases the mean carrier energy in carrier transport with an enhanced Seebeck coefficient. In addition, because the $\mathrm{C}_{60}$ nanoparticles are of zero dimensional structure, there might be quantum constraints in the carrier transport process, which would further improve the Seebeck coefficient. Ultimately, a largely enhanced Seebeck coefficient results in a synergistic effect on the thermoelectric properties.

Moreover, the incorporation of two or more kinds of filler into PEDOT:PSS can effectively optimize the thermoelectric properties. For example, Kim and co-workers ${ }^{185}$ combined the merits of both inorganic Te nanowires and carbon nanostructure fillers (rGO). Free-standing rGO/PEDOT:PSS/TeNW composite paper was prepared via mixing and then by the vacuum-filtering method and $\mathrm{HI}$ treatment. It possesses an ultrahigh power factor of $143 \mu \mathrm{W} \mathrm{m}^{-1} \mathrm{~K}^{-2}$, which is higher than that of binary hybrid materials ( $\mathrm{rGO} / \mathrm{TeNW}$ hybrid, PEDOT:PSS/ TeNW hybrid). The hybrid paper demonstrated a porous structure with a hierarchically layered morphology consisting of rGO and TeNWs. And rGO layers were coated on the surface of TeNW. The high electrical conductivity (3496 S m$~^{-1}$ ) was attributed to the graphitization of GO to rGO during the HI treatment process. Meanwhile, an enhanced Seebeck coefficient was obtained due to the synergetic energy-filtering effects at the two interfaces of PEDOT:PSS/TeNW and rGO/Te. Similar studies on composites such as SWCNTs/PEDOT:PSS coated Te nanorods, ${ }^{187}$ PEDOT:PSS/graphene/CNT ternary composites, ${ }^{188}$ and Te/PEDOT:PSS/small bundle SWCNTs composites ${ }^{186}$ have also been reported. Table 4 compares the thermoelectric properties of PEDOT:PSS based composites. It can be concluded that 1D Te filler and SWCNTs are two of the best candidates for PEDOT:PSS based thermoelectric materials. The combination of these two fillers in PEDOT:PSS could achieve the highest 
Table 4 Comparison of thermoelectric properties of PEDOT:PSS based composites

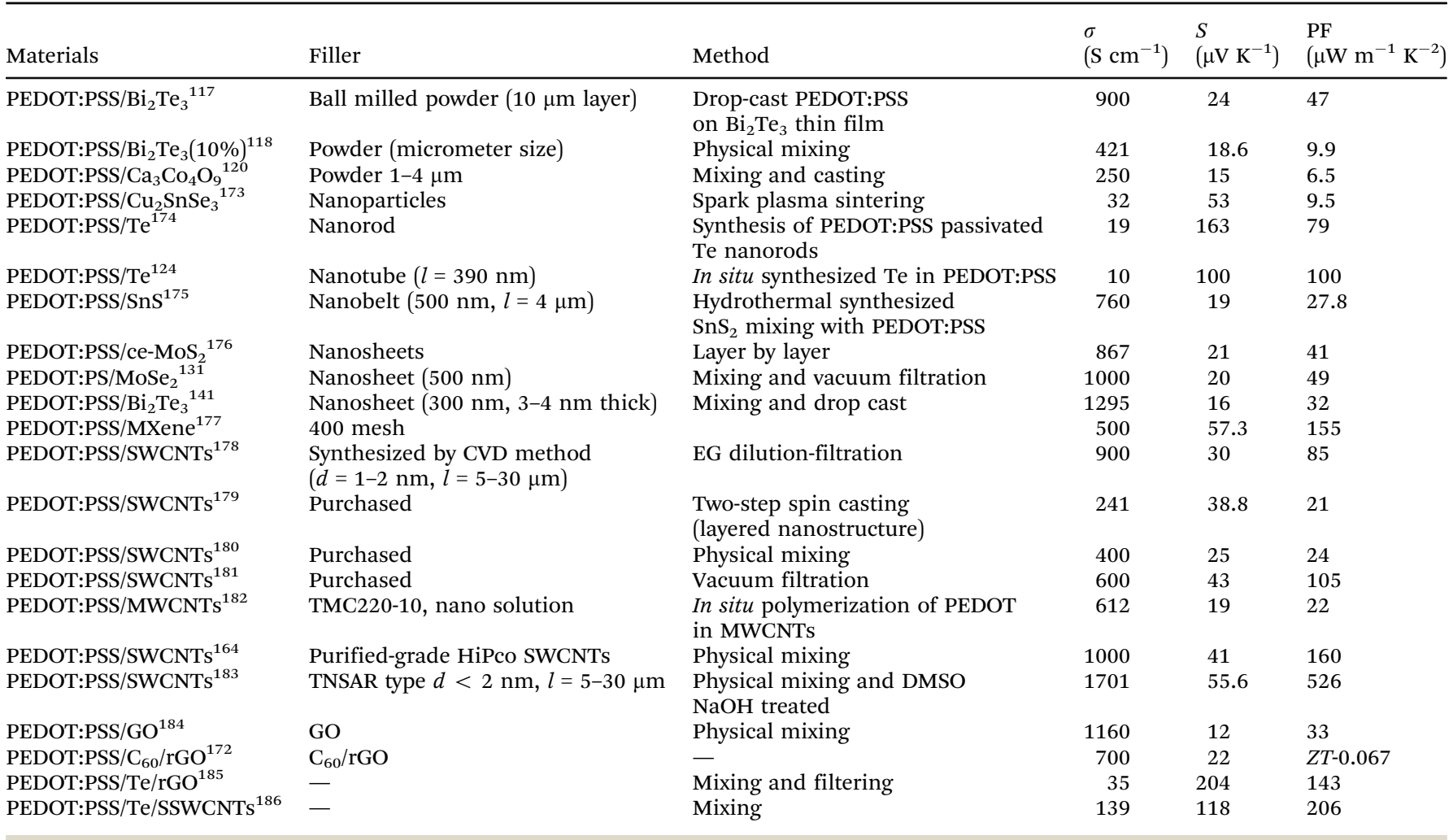

properties because of the double interfacial energy-filtering effect and tunable carrier concentration.

\section{Conclusion and outlook}

In summary, PEDOT:PSS is currently the most successful conducting polymer with high electrical conductivity, flexibility, and processability. And very often, its properties need to be tailored to suit a specific application. This paper reviews recent progress on optimizing the properties of PEDOT:PSS based blends and composites via a blending or mixing method. Miscible and immiscible polymer blends based on PEDOT:PSS exhibit striking merits such as adjustable conductivity and stretchability, and can be potentially applied in the field of flexible electronics such as in flexible electrodes, stretchable conductors and deformation sensors. Other than flexible/ stretchable electronics, PEDOT:PSS composites with incorporated inorganic and carbon fillers exhibit good thermoelectric properties (including high electrical conductivity, high Seebeck coefficient, and low thermal conductivity). These materials have demonstrated promising applications as flexible thermoelectric materials.

For blends containing small molecules, these small molecules might seep out during subsequent application, thus there are few studies carried out on this topic. For miscible polymer blends, relatively high electrical conductivity could be obtained. However, due to the plasticity of these insulating soft materials and rather poor mechanical properties of PEDOT:PSS, reversible tensile properties cannot be achieved for these materials. Meanwhile, since these insulating polymers are miscible with PEDOT:PSS, they could wrap the PEDOT colloidal particles, and thus the formation of a PEDOT conductive network might be prohibited. For immiscible polymer blends, careful attention needs to be paid on the interfacial interaction between PEDOT: PSS and the additional polymer. With the right choice of compatibilizers, strain at break exceeding $100 \%$ while maintaining a relatively high conductivity of around $70 \mathrm{~S} \mathrm{~cm}^{-1}$ could be observed. As for PEDOT:PSS based composites for thermoelectric applications, SWCNTs are found to be the best filler for this type of composite (Table 4). Meanwhile, the dimension of the filler is found to have some degree of influence on the final properties as certain type of 1D filler (Te nanorods) could induce a highly ordered PEDOT:PSS layer which may promote the carrier transport in the composites. Nevertheless, there are still some challenges that need to be further addressed for these blends and composites.

First of all, regarding the flexible PEDOT:PSS blends, the enhanced stretchablility is often associated with sacrificed electrical conductivity, despite the fact that a secondary doping method is used. In particular for immiscible polymer/PEDOT: PSS blends, due to the incompatibility between the elastomer and PEDOT:PSS, it is often complicated to prepare bulk PEDOT: PSS conductors. Besides blend fibers, there are very few research studies on constructing hierarchical structures or nano confined PEDOT:PSS in elastic matrixes to form conductive pathways since the finite-size effect and the interfacial effect are able to result in peculiar thermodynamic and kinetic 
properties. It is thought that more careful control on the processing strategy and interfacial interaction could result in extensive phase morphologies with better properties.

Secondary, in the cases of thermoelectric materials based on PEDOT:PSS, there is a large gap in figures of merit between PEDOT:PSS composites and bulk inorganic thermoelectric materials due to the conflict between electrical conductivity, Seebeck coefficient and thermal conductivity. To improve the thermoelectric properties of PEDOT:PSS based composites, first of all, it is still a great challenge to establish the theory and model of inorganic TE materials, which can predict their electrical and thermal transport properties. More work needs to be done on PEDOT:PSS as an organic TE material, such as increasing the crystallinity of PEDOT chains, controlling the morphology (ratio between PEDOT and PSS) and doping level of neat PEDOT:PSS in the synthesis or secondary doping stage that can achieve a balance of its thermoelectric properties. Secondly, based on the above discussion, the energy filtering effect and interfacial ordering effect of PEODOT:PSS nanocomposites may lead to both high Seebeck coefficient and high electrical conductivity. However, incorporating inorganic and carbon TE fillers into PEDOT:PSS may cause agglomeration and inhomogeneity, thus hindering further enhancement of the power factor. In order to achieve a uniform distribution of fillers in PEDOT:PSS, continuous efforts are required to improve the fabrication process (such as surface modulation). In addition to filler distribution, controlling the interfacial interaction and orientation of the filler should be taken into account to optimize the thermoelectric properties in future studies. Moreover, the mechanism responsible for the enhanced thermoelectric properties should also be further investigated.

Thirdly, regarding the relationship between stretchability and thermoelectrics, self-powered electronics should be explored for the application of modified PEDOT:PSS blends and composites. Once there is a temperature gradient in thermoelectric materials, thermoelectric power can be generated continuously without any moving part. There have been several studies on the preparation of flexible wearable thermoelectric generators using inorganic semiconductors based on the Seebeck effect. ${ }^{189-191}$ However, toxicity, brittleness, difficulty in processing and high cost of inorganic semiconductors limited their applications. PEDOT:PSS, with its unique flexibility, lightness and nontoxicity, has potential for self-powered devices, especially for applications in wearable and implantable electronics. At present, there are very few studies on the preparation of self-powered flexible wearable electronics using PEDOT:PSS composites based on the Seebeck effect. For instance, flexible self-powered midinfrared detectors, ${ }^{192}$ self-powered pressure sensors, ${ }^{193,194}$ selfpowered touch sensors, ${ }^{195}$ and self-powered strain sensor ${ }^{108}$ have been developed based on PEDOT:PSS composites. However, since PEDOT:PSS lacks stretchability, elastomers are often used to enhance the stretchability. Nevertheless, the thermoelectric properties of PEDOT:PSS based polymer blends are often damaged by the addition of elastomers. A balance needs to be achieved between strechability and thermoelectric properties to fabricate self-powered strain sensors. Moreover, an unavoidable problem in this application is how to ensure the stability of thermoelectric voltage under deformation (strain, bending, press and twist). Theoretically speaking, during the deformation process, the conductivity and thermoelectric properties of the composite would be changed to some extent. Up to now, the influence of deformation on the thermoelectric properties of materials has been barely studied in the literature. Therefore, utilizing thermoelectric voltage for self-powered flexible electronics is still in its infancy.

\section{Conflicts of interest}

There are no conflicts to declare.

\section{Acknowledgements}

We gratefully acknowledge the National Natural Science Foundation of China (51922071, 51773139) for financial support. This work was also supported by the Fundamental Research Funds for Central Universities.

\section{References}

1 J. Y. Lee, S. T. Connor, Y. Cui and P. Peumans, SolutionProcessed Metal Nanowire Mesh Transparent Electrodes, Nano Lett., 2018, 8, 689-692.

2 S. Park, M. Vosguerichian and Z. Bao, A review of fabrication and applications of carbon nanotube film-based flexible electronics, Nanoscale, 2013, 5, 1727-1752.

3 D. Janas, Towards monochiral carbon nanotubes: a review of progress in the sorting of single-walled carbon nanotubes, Mater. Chem. Front., 2018, 2, 36-63.

4 T.-H. Han, H. Kim, S.-J. Kwon and T.-W. Lee, Graphenebased flexible electronic devices, Mater. Sci. Eng., R, 2017, 118, 1-43.

5 M. Zhang, C. Hou, A. Halder, H. Wang and Q. Chi, Graphene papers: smart architecture and specific functionalization for biomimetics, electrocatalytic sensing and energy storage, Mater. Chem. Front., 2017, 1, 37-60.

6 Q. Wu, Y. Xu, Z. Yao, A. Liu and G. Shi, Supercapacitors based on flexible graphene/polyaniline nanofiber composite films, ACS Nano, 2010, 4, 1963-1970.

7 H. Peng, Aligned carbon nanotube/polymer composite films with robust flexibility, high transparency, and excellent conductivity, J. Am. Chem. Soc., 2008, 130, 42-43.

8 G. Tang, Z.-G. Jiang, X. Li, H.-B. Zhang and Z.-Z. Yu, Simultaneous functionalization and reduction of graphene oxide with polyetheramine and its electrically conductive epoxy nanocomposites, Chin. J. Polym. Sci., 2014, 32, 975-985.

9 P.-C. Wang and A. G. MacDiarmid, Integration of polymerdispersed liquid crystal composites with conducting polymer thin films toward the fabrication of flexible display devices, Displays, 2007, 28, 101-104.

10 W. Meixiang, Studies on Enhanced Conductivity of Stretched Conducting Polymers, Chin. J. Polym. Sci., 1995, 13, 1-6. 
11 H. Naarmann and N. Theophilou, New process for the production of metal-like, stable polyacetylene, Synth. Met., 1987, 22, 1-8.

12 M. Hughes, G. Z. Chen, M. S. P. Shaffer, D. J. Fray and A. H. Windle, Controlling the nanostructure of electrochemically grown nanoporous composites of carbon nanotubes and conducting polymers, Compos. Sci. Technol., 2004, 64, 2325-2331.

13 I. S. Chronakis, S. Grapenson and A. Jakob, Conductive polypyrrole nanofibers via electrospinning: electrical and morphological properties, Polymer, 2006, 47, 1597-1603.

14 S. R. Sivakkumar and R. Saraswathi, Performance evaluation of poly(N-methylaniline) and polyisothianaphthene in charge-storage devices, J. Power Sources, 2004, 137, 322-328.

15 Z. Yang, J. Ma, B. Bai, A. Qiu, D. Losic, D. Shi and M. Chen, Free-standing PEDOT/polyaniline conductive polymer hydrogel for flexible solid-state supercapacitors, Electrochim. Acta, 2019, 322, DOI: 10.1016/j.electacta.2019. 134769.

16 B. L. Groenendaal, F. Jonas, D. Freitag, H. Pielartzik and J. R. Reynolds, PEDOT and its derivatives; past, present, and future, Adv. Mater., 2000, 7, 481-494.

17 M. N. Spiteri, C. E. Williams and F. Boue, Pearl-necklacelike chain conformation of hydrophobic polyelectrolyte: a SANS study of partially sulfonated polystyrene in water, Macromolecules, 2007, 40, 6679-6691.

18 X. Crispin, F. Jakobsson, A. Crispin, P. Grim, P. Andersson, A. Volodin, C. Van Haesendonck, M. Van der Auweraer, W. R. Salaneck and M. Berggren, The origin of the high conductivity of poly (3, 4-ethylenedioxythiophene)-poly (styrenesulfonate)(PEDOT-PSS) plastic electrodes, Chem. Mater., 2006, 18, 4354-4360.

19 G. Greczynski, T. Kugler and W. Salaneck, Characterization of the PEDOT-PSS system by means of X-ray and ultraviolet photoelectron spectroscopy, Thin Solid Films, 1999, 354, 129-135.

20 M. Kemerink, S. Timpanaro, M. De Kok, E. Meulenkamp and F. Touwslager, Three-dimensional inhomogeneities in PEDOT: PSS films, J. Phys. Chem. B, 2004, 108, 18820-18825.

21 U. Lang, E. Müller, N. Naujoks and J. Dual, Microscopical investigations of PEDOT:PSS thin films, Adv. Funct. Mater., 2009, 19, 1215-1220.

22 J. Rivnay, S. Inal, B. A. Collins, M. Sessolo, E. Stavrinidou, X. Strakosas, C. Tassone, D. M. Delongchamp and G. G. Malliaras, Structural control of mixed ionic and electronic transport in conducting polymers, Nat. Commun., 2016, 7, 1-9.

23 D. A. Mengistie, P.-C. Wang and C.-W. Chu, Effect of molecular weight of additives on the conductivity of PEDOT: PSS and efficiency for ITO-free organic solar cells, J. Mater. Chem. A, 2013, 1, 9907-9915.

24 T. Takano, H. Masunaga, A. Fujiwara, H. Okuzaki and T. Sasaki, PEDOT nanocrystal in highly conductive PEDOT: PSS polymer films, Macromolecules, 2012, 45, 3859-3865.
25 A. M. Nardes, R. A. Janssen and M. Kemerink, A morphological model for the solvent-enhanced conductivity of PEDOT: PSS thin films, Adv. Funct. Mater., 2008, 18, 865-871.

26 Y. H. Kim, C. Sachse, M. L. Machala, C. May, L. MüllerMeskamp and K. Leo, Highly conductive PEDOT: PSS electrode with optimized solvent and thermal post-treatment for ITO-free organic solar cells, Adv. Funct. Mater., 2011, 21, 1076-1081.

27 F. Wu, P. Li, K. Sun, Y. Zhou and J. Ouyang, Conductivity Enhancement of PEDOT:PSS via Addition of Chloroplatinic Acid and Its Mechanism, Adv. Electron. Mater., 2017, 3, 1700047.

28 C. Wang, K. Sun, J. Fu, R. Chen, M. Li, Z. Zang, X. Liu, B. Li, H. Gong and J. Ouyang, Enhancement of Conductivity and Thermoelectric Property of PEDOT:PSS via Acid Doping and Single Post-Treatment for Flexible Power Generator, Adv. Sustainable Syst., 2018, 2, 1800085.

29 L. Zhang, K. Yang, R. Chen, Y. Zhou, S. Chen, Y. Zheng, M. Li, C. Xu, X. Tang and Z. Zang, The role of mineral acid doping of PEDOT: PSS and its application in organic photovoltaics, Adv. Electron. Mater., 2020, 6, 1900648.

30 J. Ouyang, Q. Xu, C.-W. Chu, Y. Yang, G. Li and J. Shinar, On the mechanism of conductivity enhancement in poly (3, 4-ethylenedioxythiophene): poly (styrene sulfonate) film through solvent treatment, Polymer, 2004, 45, 8443-8450.

31 N. Kim, S. Kee, S. H. Lee, B. H. Lee, Y. H. Kahng, Y. R. Jo, B. J. Kim and K. Lee, Highly conductive PEDOT: PSS nanofibrils induced by solution-processed crystallization, Adv. Mater., 2014, 26, 2268-2272.

32 H. Shi, C. Liu, Q. Jiang and J. Xu, Effective approaches to improve the electrical conductivity of PEDOT:PSS: a review, Adv. Electron. Mater., 2015, 1, 1500017.

33 J. Ouyang, Secondary doping methods to significantly enhance the conductivity of PEDOT: PSS for its application as transparent electrode of optoelectronic devices, Displays, 2013, 34, 423-436.

34 J. Ouyang, Recent advances of intrinsically conductive polymers, Acta Phys.-Chim. Sin., 2018, 34, 1211-1220.

35 S. Bi, X. Leng, Y. Li, Z. Zheng, X. Zhang, Y. Zhang and H. Zhou, Interfacial modification in organic and perovskite solar cells, Adv. Mater., 2019, 31, 1805708.

36 X. Fan, W. Song, T. Lei, B. Xu, F. Yan, N. Wang, H. Cui and Z. Ge, High-efficiency robust organic solar cells using transfer-printed PEDOT: PSS electrodes through interface bonding engineering, Mater. Chem. Front., 2019, 3, 901-908.

37 C. Yang, Z. Sun, Y. He, B. Xiong, S. Chen, M. Li, Y. Zhou, Y. Zheng and K. Sun, Performance-enhancing Approaches for the PEDOT: PSS-Si Hybrid Solar Cells, Angew. Chem., 2019, DOI: 10.1002/ange.201910629.

38 G. Greczynski, T. Kugler, M. Keil, W. Osikowicz, M. Fahlman and W. R. Salaneck, Photoelectron spectroscopy of thin films of PEDOT-PSS conjugated polymer blend: a mini-review and some new results, J. Electron Spectrosc. Relat. Phenom., 2001, 121, 1-17. 
39 S. Ho, S. Liu, Y. Chen and F. So, Review of recent progress in multilayer solution-processed organic light-emitting diodes, J. Photonics Energy, 2015, 5, 057611.

40 Z. Zhao, G. F. Richardson, Q. Meng, S. Zhu, H.-C. Kuan and J. Ma, PEDOT-based composites as electrode materials for supercapacitors, Nanotechnology, 2016, 27, 042001.

41 Y. Gao, Graphene and polymer composites for supercapacitor applications: a review, Nanoscale Res. Lett., 2017, 12, 387.

42 T. Cheng, Y.-Z. Zhang, J.-D. Zhang, W.-Y. Lai and W. Huang, High-performance free-standing PEDOT: PSS electrodes for flexible and transparent all-solid-state supercapacitors, J. Mater. Chem. A, 2016, 4(27), 10493-10499.

$43 \mathrm{R}$. Yue and J. Xu, Poly (3, 4-ethylenedioxythiophene) as promising organic thermoelectric materials: A minireview, Synth. Met., 2012, 162, 912-917.

44 Z. Zhu, C. Liu, F. Jiang, J. Xu and E. Liu, Effective treatment methods on PEDOT: PSS to enhance its thermoelectric performance, Synth. Met., 2017, 225, 31-40.

45 M. Culebras, C. M. Gómez and A. Cantarero, Review on polymers for thermoelectric applications, Materials, 2014, 7, 6701-6732.

46 D. J. Lipomi, Stretchable figures of merit in deformable electronics, Adv. Mater., 2016, 28, 4180-4183.

47 D. J. Lipomi and Z. Bao, Stretchable and ultraflexible organic electronics, MRS Bull., 2017, 42, 93-97.

48 S. E. Root, S. Savagatrup, A. D. Printz, D. Rodriquez and D. J. Lipomi, Mechanical properties of organic semiconductors for stretchable, highly flexible, and mechanically robust electronics, Chem. Rev., 2017, 117, 6467-6499.

49 X. Fan, W. Nie, H. Tsai, N. Wang, H. Huang, Y. Cheng, R. Wen, L. Ma, F. Yan and Y. Xia, PEDOT: PSS for flexible and stretchable electronics: modifications, strategies, and applications, Adv. Sci., 2019, 6, 1900813.

50 L. V. Kayser and D. J. Lipomi, Stretchable conductive polymers and composites based on PEDOT and PEDOT: PSS, Adv. Mater., 2019, 31, 1806133.

51 D. H. Kim, J. Xiao, J. Song, Y. Huang and J. A. Rogers, Stretchable, curvilinear electronics based on inorganic materials, Adv. Mater., 2010, 22, 2108-2124.

52 E. Roh, B.-U. Hwang, D. Kim, B.-Y. Kim and N.-E. Lee, Stretchable, transparent, ultrasensitive, and patchable strain sensor for human-machine interfaces comprising a nanohybrid of carbon nanotubes and conductive elastomers, ACS Nano, 2015, 9, 6252-6261.

53 X. Fan, N. Wang, J. Wang, B. Xu and F. Yan, Highly sensitive, durable and stretchable plastic strain sensors using sandwich structures of PEDOT: PSS and an elastomer, Mater. Chem. Front., 2018, 2, 355-361.

54 I. Põldsalu, M. Harjo, T. Tamm, M. Uibu, A.-L. Peikolainen and R. Kiefer, Inkjet-printed hybrid conducting polymeractivated carbon aerogel linear actuators driven in an organic electrolyte, Sens. Actuators, B, 2017, 250, 44-51.

55 F. Zabihi, Y. Xie, S. Gao and M. Eslamian, Morphology, conductivity, and wetting characteristics of PEDOT: PSS thin films deposited by spin and spray coating, Appl. Surf. Sci., 2015, 338, 163-177.
56 J. Suikkola, T. Björninen, M. Mosallaei, T. Kankkunen, P. Iso-Ketola, L. Ukkonen, J. Vanhala and M. Mäntysalo, Screen-printing fabrication and characterization of stretchable electronics, Sci. Rep., 2016, 6, 25784.

57 Y. Wang, C. Zhu, R. Pfattner, H. Yan, L. Jin, S. Chen, F. Molina-Lopez, F. Lissel, J. Liu and N. I. Rabiah, A highly stretchable, transparent, and conductive polymer, Sci. Adv., 2017, 3, e1602076.

58 N. M. Nair, J. K. Pakkathillam, K. Kumar, K. Arunachalam, D. Ray and P. Swaminathan, A printable silver nanowire and PEDOT: PSS nanocomposite ink for flexible transparent conducting applications, ACS Appl. Electron. Mater., 2020, DOI: 10.1021/acsaelm.0c00061.

59 J. G. Tait, B. J. Worfolk, S. A. Maloney, T. C. Hauger, A. L. Elias, J. M. Buriak and K. D. Harris, Spray coated high-conductivity PEDOT: PSS transparent electrodes for stretchable and mechanically-robust organic solar cells, Sol. Energy Mater. Sol. Cells, 2013, 110, 98-106.

60 M. Vosgueritchian, D. J. Lipomi and Z. Bao, Highly conductive and transparent PEDOT: PSS films with a fluorosurfactant for stretchable and flexible transparent electrodes, Adv. Funct. Mater., 2012, 22, 421-428.

61 D. J. Lipomi, J. A. Lee, M. Vosgueritchian, B. C.-K. Tee, J. A. Bolander and Z. Bao, Electronic properties of transparent conductive films of PEDOT: PSS on stretchable substrates, Chem. Mater., 2012, 24, 373-382.

62 Z. Wen, Y. Yang, N. Sun, G. Li, Y. Liu, C. Chen, J. Shi, L. Xie, H. Jiang and D. Bao, A wrinkled PEDOT: PSS film based stretchable and transparent triboelectric nanogenerator for wearable energy harvesters and active motion sensors, Adv. Funct. Mater., 2018, 28, 1803684.

63 S. K. Nemani, D. Chen, M. H. Mohamed and H. Sojoudi, Stretchable and hydrophobic electrochromic devices using wrinkled graphene and PEDOT:PSS, J. Nanomater., 2018, 2018, 1-10.

64 B. Sarkar, D. K. Satapathy and M. Jaiswal, Wrinkle and crack-dependent charge transport in a uniaxially strained conducting polymer film on a flexible substrate, Soft Matter, 2017, 13, 5437-5444.

65 Z. Zhao, M. Moussa, G. Shi, Q. Meng, R. Wang and J. Ma, Compressible, electrically conductive, fibre-like, threedimensional PEDOT-based composite aerogels towards energy storage applications, Compos. Sci. Technol., 2016, 127, 36-46.

66 K. Sun, S. Zhang, P. Li, Y. Xia, X. Zhang, D. Du, F. H. Isikgor and J. Ouyang, Review on application of PEDOTs and PEDOT:PSS in energy conversion and storage devices, J. Mater. Sci.: Mater. Electron., 2015, 26, 4438-4462.

67 U. Lang and J. Dual, Mechanical Properties of the Intrinsically Conductive Polymer Poly(3,4- Ethylenedioxythiophene) Poly(Styrenesulfonate) (PEDOT/PSS), Key Eng. Mater., 2007, 1189-1192.

68 U. Lang, N. Naujoks and J. Dual, Mechanical characterization of PEDOT:PSS thin films, Synth. Met., 2009, 159, 473-479.

69 S. R. Dupont, F. Novoa, E. Voroshazi and R. H. Dauskardt, Decohesion kinetics of PEDOT:PSS conducting polymer films, Adv. Funct. Mater., 2014, 24, 1325-1332. 
70 J. Zhou, D. H. Anjum, L. Chen, X. Xu, I. A. Ventura, L. Jiang and G. Lubineau, The temperature-dependent microstructure of PEDOT/PSS films: insights from morphological, mechanical and electrical analyses, J. Mater. Chem. C, 2014, 2, 9903-9910.

71 J. M. G. Cowie and V. Arrighi, Polymers: chemistry and physics of modern materials, CRC Press, 2007.

72 T. Stöcker, A. Köhler and R. Moos, Why does the electrical conductivity in PEDOT: PSS decrease with PSS content? A study combining thermoelectric measurements with impedance spectroscopy, J. Polym. Sci., Part B: Polym. Phys., 2012, 50, 976-983.

73 Y. Li, R. Tanigawa and H. Okuzaki, Soft and flexible PEDOT/PSS films for applications to soft actuators, Smart Mater. Struct., 2014, 23, 074010.

74 Y. Li, Y. Masuda, Y. Iriyama and H. Okuzaki, Stretchable and highly conductive polymer films, Trans. Mater. Res. Soc. Jpn., 2012, 37, 303-306.

75 S.-S. Yoon and D.-Y. Khang, Roles of nonionic surfactant additives in PEDOT: PSS thin films, J. Phys. Chem. C, 2016, 120, 29525-29532.

76 S. Savagatrup, E. Chan, S. M. Renteria-Garcia, A. D. Printz, A. V. Zaretski, T. F. O'Connor, D. Rodriquez, E. Valle and D. J. Lipomi, Plasticization of PEDOT: PSS by common additives for mechanically robust organic solar cells and wearable sensors, Adv. Funct. Mater., 2015, 25, 427-436.

77 J. Y. Oh, S. Kim, H. K. Baik and U. Jeong, Conducting polymer dough for deformable electronics, Adv. Mater., 2016, 28, 4455-4461.

78 M. Y. Teo, N. Kim, S. Kee, B. S. Kim, G. Kim, S. Hong, S. Jung and K. Lee, Highly stretchable and highly conductive PEDOT: PSS/ionic liquid composite transparent electrodes for solution-processed stretchable electronics, ACS Appl. Mater. Interfaces, 2017, 9, 819-826.

79 S. Kee, H. Kim, S. H. K. Paleti, A. El Labban, M. Neophytou, A.-H. Emwas, H. N. Alshareef and D. Baran, Highly Stretchable and Air-Stable PEDOT: PSS/Ionic Liquid Composites for Efficient Organic Thermoelectrics, Chem. Mater., 2019, 31, 3519-3526.

80 M. Döbbelin, R. Marcilla, M. Salsamendi, C. Pozo-Gonzalo, P. M. Carrasco, J. A. Pomposo and D. Mecerreyes, Influence of ionic liquids on the electrical conductivity and morphology of PEDOT: PSS films, Chem. Mater., 2007, 19, 2147-2149.

81 D.-P. Lin, H.-W. He, Y.-Y. Huang, W.-P. Han, G.-F. Yu, X. Yan, Y.-Z. Long and L.-H. Xia, Twisted microropes for stretchable devices based on electrospun conducting polymer fibers doped with ionic liquid, J. Mater. Chem. C, 2014, 2, 8962-8966.

82 P. Li, K. Sun and J. Ouyang, Stretchable and Conductive polymer films prepared by solution blending, ACS Appl. Mater. Interfaces, 2015, 7, 18415-18423.

83 A. R. Hopkins and J. R. Reynolds, Crystallization driven formation of conducting polymer networks in polymer blends, Macromolecules, 2000, 33, 5221-5226.

84 N. Liu, G. Fang, J. Wan, H. Zhou, H. Long and X. Zhao, Electrospun PEDOT: PSS-PVA nanofiber based ultrahigh-strain sensors with controllable electrical conductivity, J. Mater. Chem., 2011, 21, 18962-18966.

85 C.-h. Chen, A. Torrents, L. Kulinsky, R. D. Nelson, M. J. Madou, L. Valdevit and J. C. LaRue, Mechanical characterizations of cast Poly (3, 4-ethylenedioxythiophene): Poly (styrenesulfonate)/Polyvinyl Alcohol thin films, Synth. Met., 2011, 161, 2259-2267.

86 A. Rinaldi, R. Matos, A. Rubira, O. Ferreira and E. Girotto, Electrical, spectroscopic, and thermal properties of blends formed by PEDOT, PVC, and PEO, J. Appl. Polym. Sci., 2005, 96, 1710-1715.

87 K. Fu, R. Lv, B. Na, S. Zou, R. Zeng, B. Wang and H. Liu, Mixed ion-electron conducting PEO/PEDOT: PSS miscible blends with intense electrochromic response, Polymer, 2019, 184, 121900.

88 J. H. Lee, Y. R. Jeong, G. Lee, S. W. Jin, Y. H. Lee, S. Y. Hong, H. Park, J. W. Kim, S.-S. Lee and J. S. Ha, Highly conductive, stretchable, and transparent PEDOT: PSS electrodes fabricated with triblock copolymer additives and acid treatment, ACS Appl. Mater. Interfaces, 2018, 10, 28027-28035.

89 P. Li, D. Du, L. Guo, Y. Guo and J. Ouyang, Stretchable and conductive polymer films for high-performance electromagnetic interference shielding, J. Mater. Chem. C, 2016, 4, 6525-6532.

90 J. Zhou, G. Tian, G. Jin, Y. Xin, R. Tao and G. Lubineau, Buckled Conductive Polymer Ribbons in Elastomer Channels as Stretchable Fiber Conductor, Adv. Funct. Mater., 2019, 1907316.

91 G. Tian, J. Zhou, Y. Xin, R. Tao, G. Jin and G. Lubineau, Copolymer-enabled stretchable conductive polymer fibers, Polymer, 2019, 177, 189-195.

92 C. M. Palumbiny, F. Liu, T. P. Russell, A. Hexemer, C. Wang and P. Müller-Buschbaum, The crystallization of PEDOT: PSS polymeric electrodes probed in situ during printing, Adv. Mater., 2015, 27, 3391-3397.

93 J. Zhou, E. Q. Li, R. Li, X. Xu, I. A. Ventura, A. Moussawi, D. H. Anjum, M. N. Hedhili, D.-M. Smilgies and G. Lubineau, Semi-metallic, strong and stretchable wet-spun conjugated polymer microfibers, J. Mater. Chem. C, 2015, 3, 2528-2538.

94 X. Li, Y. Liu, Z. Shi, C. Li and G. Chen, Influence of draw ratio on the structure and properties of PEDOT-PSS/PAN composite conductive fibers, $R S C A d v$., 2014, 4, 40385-40389.

95 Q. Gao, M. Wang, X. Kang, C. Zhu and M. Ge, Continuous wet-spinning of flexible and water-stable conductive PEDOT: PSS/PVA composite fibers for wearable sensors, Compos. Commun., 2020, 17, 134-140.

96 H. He, L. Zhang, X. Guan, H. Cheng, X. Liu, S. Yu, J. Wei and J. Ouyang, Biocompatible conductive polymers with high conductivity and high stretchability, ACS Appl. Mater. Interfaces, 2019, 11, 26185-26193.

97 P. Li, Y. Wang, U. Gupta, J. Liu, L. Zhang, D. Du, C. C. Foo, J. Ouyang and J. Zhu, Transparent Soft Robots for Effective Camouflage, Adv. Funct. Mater., 2019, 29, 1901908. 
98 J.-S. Noh, Highly conductive and stretchable poly (dimethylsiloxane): poly (3, 4-ethylenedioxythiophene): poly (styrene sulfonic acid) blends for organic interconnects, RSC Adv., 2014, 4, 1857-1863.

99 R. Luo, H. Li, B. Du, S. Zhou and Y. Zhu, A simple strategy for high stretchable, flexible and conductive polymer films based on PEDOT: PSS-PDMS blends, Org. Electron., 2020, 76, 105451.

100 C. Teng, X. Lu, Y. Zhu, M. Wan and L. Jiang, Polymer in situ embedding for highly flexible, stretchable and water stable PEDOT: PSS composite conductors, RSC Adv., 2013, 3, 7219-7223.

101 Y. Ko, D. Kim, U.-J. Kim and J. You, Vacuum-assisted bilayer PEDOT: PSS/cellulose nanofiber composite film for self-standing, flexible, conductive electrodes, Carbohydr. Polym., 2017, 173, 383-391.

102 M. Lay, M. À. Pèlach, N. Pellicer, J. A. Tarrés, K. N. Bun and F. Vilaseca, Smart nanopaper based on cellulose nanofibers with hybrid PEDOT: PSS/polypyrrole for energy storage devices, Carbohydr. Polym., 2017, 165, 86-95.

103 S. Khan, M. Ul-Islam, W. A. Khattak, M. W. Ullah and J. K. Park, Bacterial cellulose-poly (3, 4-ethylenedioxythiophene)-poly (styrenesulfonate) composites for optoelectronic applications, Carbohydr. Polym., 2015, 127, 86-93.

104 K. M. Alam, P. Kar, U. K. Thakur, R. Kisslinger, N. Mahdi, A. Mohammadpour, P. A. Baheti, P. Kumar and K. Shankar, Remarkable self-organization and unusual conductivity behavior in cellulose nanocrystal-PEDOT: PSS nanocomposites, J. Mater. Sci.: Mater. Electron., 2019, 30, 1390-1399.

105 J. Zhou and Y.-L. Hsieh, Conductive polymer protonated nanocellulose aerogels for tunable and linearly responsive strain sensors, ACS Appl. Mater. Interfaces, 2018, 10, 27902-27910.

106 H.-E. Yin, C.-H. Wu, K.-S. Kuo, W.-Y. Chiu and H.-J. Tai, Innovative elastic and flexible conductive PEDOT: PSS composite films prepared by introducing soft latexes, J. Mater. Chem., 2012, 22, 3800-3810.

107 Z. Wang, T. Wang, M. Zhuang and H. Xu, Stretchable Polymer Composite with a 3D Segregated Structure of PEDOT: PSS for Multifunctional Touchless Sensing, ACS Appl. Mater. Interfaces, 2019, 11, 45301-45309.

108 P. J. Taroni, G. Santagiuliana, K. Wan, P. Calado, M. Qiu, H. Zhang, N. M. Pugno, M. Palma, N. Stingelin-Stutzman and $\mathrm{M}$. Heeney, Toward stretchable self-powered sensors based on the thermoelectric response of PEDOT: PSS/ polyurethane blends, Adv. Funct. Mater., 2018, 28, 1704285.

109 L. V. Kayser, M. D. Russell, D. Rodriquez, S. N. Abuhamdieh, C. Dhong, S. Khan, A. N. Stein, J. Ramírez and D. J. Lipomi, RAFT Polymerization of an Intrinsically Stretchable Water-Soluble Block Copolymer Scaffold for PEDOT, Chem. Mater., 2018, 30, 4459-4468.

110 M. Z. Seyedin, J. M. Razal, P. C. Innis and G. G. Wallace, Strain-responsive polyurethane/PEDOT: PSS elastomeric composite Fibers with high electrical conductivity, Adv. Funct. Mater., 2014, 24, 2957-2966.
111 H. Song and K. Cai, Preparation and properties of PEDOT: PSS/Te nanorod composite films for flexible thermoelectric power generator, Energy, 2017, 125, 519-525.

112 O. Bubnova, Z. U. Khan, A. Malti, S. Braun, M. Fahlman, M. Berggren and X. Crispin, Optimization of the thermoelectric figure of merit in the conducting polymer poly (3, 4-ethylenedioxythiophene), Nat. Mater., 2011, 10, 429-433.

113 Z. Han and A. Fina, Thermal conductivity of carbon nanotubes and their polymer nanocomposites: A review, Prog. Polym. Sci., 2011, 36, 914-944.

114 H. Yao, Z. Fan, H. Cheng, X. Guan, C. Wang, K. Sun and J. Ouyang, Recent Development of Thermoelectric Polymers and Composites, Macromol. Rapid Commun., 2018, 1700727.

115 Y. Li, Y. Du, Y. Dou, K. Cai and J. Xu, PEDOT-based thermoelectric nanocomposites - A mini-review, Synth. Met., 2017, 226, 119-128.

116 Y. Zheng, Q. Zhang, X. Su, H. Xie, S. Shu, T. Chen, G. Tan, Y. Yan, X. Tang and C. Uher, Mechanically robust BiSbTe alloys with superior thermoelectric performance: a case study of stable hierarchical nanostructured thermoelectric materials, Adv. Energy Mater., 2015, 5, 1401391.

117 B. Zhang, J. Sun, H. Katz, F. Fang and R. Opila, Promising thermoelectric properties of commercial PEDOT: PSS materials and their Bi2Te3 powder composites, ACS Appl. Mater. Interfaces, 2010, 2, 3170-3178.

118 H. Song, C. Liu, H. Zhu, F. Kong, B. Lu, J. Xu, J. Wang and F. Zhao, Improved thermoelectric performance of freestanding PEDOT: PSS/Bi 2 Te 3 films with low thermal conductivity, J. Electron. Mater., 2013, 42, 1268-1274.

119 K. Wei, T. Stedman, Z.-H. Ge, L. M. Woods and G. S. Nolas, A synthetic approach for enhanced thermoelectric properties of PEDOT: PSS bulk composites, Appl. Phys. Lett., 2015, 107(15), 153301, DOI: 10.1063/1.4933254.

120 C. Liu, F. Jiang, M. Huang, B. Lu, R. Yue and J. Xu, Freestanding PEDOT-PSS/Ca 3 Co 4 O 9 composite films as novel thermoelectric materials, J. Electron. Mater., 2011, 40, 948-952.

121 Y. Du, K. Cai, S. Z. Shen, W. Yang, J. Xu and T. Lin, ZnO flower/PEDOT: PSS thermoelectric composite films, J. Mater. Sci.: Mater. Electron., 2016, 27, 10289-10293.

122 H. Lu, H. Song, H. Shi, Q. Jiang, L. Zhang, C. Liu and J. Xu, Preparation of poly (3, 4-ethylenedioxythiophene)-poly (styrenesulfonate)/Fe3O4 nanocomposite film and its thermoelectric performance, J. Compos. Mater., 2014, 48, 2793-2801.

123 S. Shin, J. W. Roh, H.-S. Kim and R. Chen, Role of surfactant on thermoelectric behaviors of organicinorganic composites, J. Appl. Phys., 2018, 123, 205106.

124 S. K. Yee, N. E. Coates, A. Majumdar, J. J. Urban and R. A. Segalman, Thermoelectric power factor optimization in PEDOT: PSS tellurium nanowire hybrid composites, Phys. Chem. Chem. Phys., 2013, 15, 4024-4032.

125 H. Song, K. Cai and S. Shen, Enhanced thermoelectric properties of PEDOT/PSS/Te composite films treated with H 2 SO 4, J. Nanopart. Res., 2016, 18, 386. 
126 J. Xiong, L. Wang, J. Xu, C. Liu, W. Zhou, H. Shi, Q. Jiang and F. Jiang, Thermoelectric performance of PEDOT: PSS/ Bi 2 Te 3-nanowires: a comparison of hybrid types, J. Mater. Sci.: Mater. Electron., 2016, 27, 1769-1776.

127 H. Huang, Y. Cui, Q. Li, C. Dun, W. Zhou, W. Huang, L. Chen, C. A. Hewitt and D. L. Carroll, Metallic 1T phase MoS2 nanosheets for high-performance thermoelectric energy harvesting, Nano Energy, 2016, 26, 172-179.

128 T. Wang, C. Liu, J. Xu, Z. Zhu, E. Liu, Y. Hu, C. Li and F. Jiang, Thermoelectric performance of restacked MoS2 nanosheets thin-film, Nanotechnology, 2016, 27, 285703.

129 X. Wang, F. Meng, H. Tang, Z. Gao, S. Li, F. Jiang and J. Xu, An effective dual-solvent treatment for improving the thermoelectric property of PEDOT: PSS with white graphene, J. Mater. Sci., 2017, 52, 9806-9818.

130 L.-D. Zhao, S.-H. Lo, Y. Zhang, H. Sun, G. Tan, C. Uher, C. Wolverton, V. P. Dravid and M. G. Kanatzidis, Ultralow thermal conductivity and high thermoelectric figure of merit in SnSe crystals, Nature, 2014, 508, 373-377.

131 X. Li, C. Liu, T. Wang, W. Wang, X. Wang, Q. Jiang, F. Jiang and J. Xu, Preparation of 2D MoSe2/PEDOT: PSS composite and its thermoelectric properties, Mater. Res. Express, 2017, 4, 116410.

132 Y. Du, X. Liu, J. Xu and S. Z. Shen, Flexible Bi-Te-based alloy nanosheet/PEDOT: PSS thermoelectric power generators, Mater. Chem. Front., 2019, 3, 1328-1334.

133 J.-Y. Lee and Y.-J. Lin, Effect of incorporation of black phosphorus into PEDOT: PSS on conductivity and electron-phonon coupling, Synth. Met., 2016, 212, 180-185.

134 F. Jiang, J. Xiong, W. Zhou, C. Liu, L. Wang, F. Zhao, H. Liu and $\mathrm{J}$. Xu, Use of organic solvent-assisted exfoliated MoS 2 for optimizing the thermoelectric performance of flexible PEDOT: PSS thin films, J. Mater. Chem. A, 2016, 4, 5265-5273.

135 N. E. Coates, S. K. Yee, B. McCulloch, K. C. See, A. Majumdar, R. A. Segalman and J. J. Urban, Effect of interfacial properties on polymer-nanocrystal thermoelectric transport, Adv. Mater., 2013, 25, 1629-1633.

136 Q. Meng, Q. Jiang, K. Cai and L. Chen, Preparation and thermoelectric properties of PEDOT: PSS coated Te nanorod/PEDOT: PSS composite films, Org. Electron., 2019, 64, 79-85.

137 J. Choi, J. Y. Lee, S. S. Lee, C. R. Park and H. Kim, HighPerformance Thermoelectric Paper Based on Double Carrier-Filtering Processes at Nanowire Heterojunctions, Adv. Energy Mater., 2016, 6, 1502181.

138 D. Song, M. Li, Y. Jiang, Z. Chen, F. Bai, Y. Li and B. Jiang, Facile fabrication of MoS2/PEDOT-PSS composites as lowcost and efficient counter electrodes for dye-sensitized solar cells, J. Photochem. Photobiol., A, 2014, 279, 47-51.

139 G. U. Siddiqui, M. Sajid, J. Ali, S. W. Kim, Y. H. Doh and K. H. Choi, Wide range highly sensitive relative humidity sensor based on series combination of MoS2 and PEDOT: PSS sensors array, Sens. Actuators, B, 2018, 266, 354-363.

140 Y. Ge, R. Jalili, C. Wang, T. Zheng, Y. Chao and G. G. Wallace, A robust free-standing MoS2/poly (3, 4ethylenedioxythiophene): poly (styrenesulfonate) film for supercapacitor applications, Electrochim. Acta, 2017, 235, 348-355.

141 Y. Du, K. Cai, S. Chen, P. Cizek and T. Lin, Facile preparation and thermoelectric properties of Bi2Te3 based alloy nanosheet/PEDOT: PSS composite films, ACS Appl. Mater. Interfaces, 2014, 6, 5735-5743.

142 C. Ou, A. L. Sangle, A. Datta, Q. Jing, T. Busolo, T. Chalklen, V. Narayan and S. Kar-Narayan, Fully Printed Organic-Inorganic Nanocomposites for Flexible Thermoelectric Applications, ACS Appl. Mater. Interfaces, 2018, 10(23), 19580-19587.

143 T. Zhang, K. Li, C. Li, S. Ma, H. H. Hng and L. Wei, Mechanically Durable and Flexible Thermoelectric Films from PEDOT:PSS/PVA/Bi0.5Sb1.5Te3 Nanocomposites, Adv. Electron. Mater., 2017, 3(4), 1600554.

144 N. Toshima, N. Jiravanichanun and H. Marutani, Organic thermoelectric materials composed of conducting polymers and metal nanoparticles, J. Electron. Mater., 2012, 41, 1735-1742.

145 N. Toshima and N. Jiravanichanun, Improvement of thermoelectric properties of PEDOT/PSS films by addition of gold nanoparticles: enhancement of seebeck coefficient, J. Electron. Mater., 2013, 42, 1882-1887.

146 A. Yoshida and N. Toshima, Thermoelectric properties of hybrid thin films of PEDOT-PSS and silver nanowires, J. Electron. Mater., 2016, 45, 2914-2919.

147 Y. Qingqing, Y. Jinliang and M. Delan, Transparent and conductive PEDOT: PSS/Ag NW/PEDOT: PSS hybrid films prepared by spin-coating at room temperature, J. Semicond., 2015, 36, 123005.

148 A. Yoshida and N. Toshima, Gold nanoparticle and gold nanorod embedded PEDOT: PSS thin films as organic thermoelectric materials, J. Electron. Mater., 2014, 43, 1492-1497.

149 Y. Liu, Z. Song, Q. Zhang, Z. Zhou, Y. Tang, L. Wang, J. Zhu, W. Luo and W. Jiang, Preparation of bulk AgNWs/ PEDOT: PSS composites: a new model towards highperformance bulk organic thermoelectric materials, RSC Adv., 2015, 5, 45106-45112.

150 J.-H. Lee, H.-S. Shin, Y.-J. Noh, S.-I. Na and H.-K. Kim, Brush painting of transparent PEDOT/Ag nanowire/PEDOT multilayer electrodes for flexible organic solar cells, Sol. Energy Mater. Sol. Cells, 2013, 114, 15-23.

151 Z. Yu, C. Li, D. Abbitt and J. Thomas, Flexible, sandwichlike Ag-nanowire/PEDOT: PSS-nanopillar/MnO 2 high performance supercapacitors, J. Mater. Chem. A, 2014, 2, 10923-10929.

152 J.-E. Lim, S.-M. Lee, S.-S. Kim, T.-W. Kim, H.-W. Koo and H.-K. Kim, Brush-paintable and highly stretchable Ag nanowire and PEDOT: PSS hybrid electrodes, Sci. Rep., 2017, 7, 1-12.

153 S. Kim, S. Y. Kim, J. Kim and J. H. Kim, Highly reliable AgNW/PEDOT: PSS hybrid films: efficient methods for enhancing transparency and lowering resistance and haziness, J. Mater. Chem. C, 2014, 2, 5636-5643.

154 Y.-s. Liu, J. Feng, X.-L. Ou, H.-F. Cui, M. Xu and H.-B. Sun, Ultrasmooth, highly conductive and transparent PEDOT: 
PSS/silver nanowire composite electrode for flexible organic light-emitting devices, Org. Electron., 2016, 31, 247-252.

155 S. Li, S. Chen, B. Zhuo, Q. Li, W. Liu and X. Guo, Flexible ammonia sensor based on PEDOT: PSS/silver nanowire composite film for meat freshness monitoring, IEEE Electron Device Lett., 2017, 38, 975-978.

156 C. Ou, A. L. Sangle, A. Datta, Q. Jing, T. Busolo, T. Chalklen, V. Narayan and S. Kar-Narayan, Fully printed organic-inorganic nanocomposites for flexible thermoelectric applications, ACS Appl. Mater. Interfaces, 2018, 10, 19580-19587.

157 J. Park, A. Lee, Y. Yim and E. Han, Electrical and thermal properties of PEDOT: PSS films doped with carbon nanotubes, Synth. Met., 2011, 161, 523-527.

158 W. Lee, Y. H. Kang, J. Y. Lee, K.-S. Jang and S. Y. Cho, Improving the thermoelectric power factor of CNT/PEDOT: PSS nanocomposite films by ethylene glycol treatment, RSC Adv., 2016, 6, 53339-53344.

159 Y. Zhu, N. Li, T. Lv, Y. Yao, H. Peng, J. Shi, S. Cao and T. Chen, Ag-Doped PEDOT: PSS/CNT composites for thinfilm all-solid-state supercapacitors with a stretchability of 480\%, J. Mater. Chem. A, 2018, 6, 941-947.

160 B.-J. Kim, S.-H. Han and J.-S. Park, Sheet resistance, transmittance, and chromatic property of CNTs coated with PEDOT: PSS films for transparent electrodes of touch screen panels, Thin Solid Films, 2014, 572, 68-72.

161 D. J. Beesley, B. Price, S. Hunter, M. S. Shaffer and J. De Mello, Direct dispersion of SWNTs in highly conductive solventenhanced PEDOT: PSS films, Nanocomposites, 2016, 2, 135-140.

162 T. Ebbesen, H. Lezec, H. Hiura, J. Bennett, H. Ghaemi and T. Thio, Electrical conductivity of individual carbon nanotubes, Nature, 1996, 382, 54-56.

163 M. F. De Volder, S. H. Tawfick, R. H. Baughman and A. J. Hart, Carbon nanotubes: present and future commercial applications, Science, 2013, 339, 535-539.

164 C. Yu, K. Choi, L. Yin and J. C. Grunlan, Light-Weight Flexible Carbon Nanotube Based Organic Composites with Large Thermoelectric Power Factors, ACS Nano, 2011, 5, 7885-7892.

165 Q. Jiang, X. Lan, C. Liu, H. Shi, Z. Zhu, F. Zhao, J. Xu and F. Jiang, High-performance hybrid organic thermoelectric SWNTS/PEDOT: PSS thin-films for energy harvesting, Mater. Chem. Front., 2018, 2, 679-685.

166 B.-J. Kim, S.-H. Han and J.-S. Park, Properties of CNTs coated by PEDOT: PSS films via spin-coating and electrophoretic deposition methods for flexible transparent electrodes, Surf. Coat. Technol., 2015, 271, 22-26.

167 H. Cheng, L. Medina and H. Duong, Free-standing PEDOT: PSS/CNT aerogels and their electrochemical performance, Mater. Technol., 2017, 32, 622-629.

168 T. M. Tritt, H. Böttner and L. Chen, Thermoelectrics: Direct solar thermal energy conversion, MRS Bull., 2008, 33, 366-368.

169 C. Jacoboni, C. Canali, G. Ottaviani and A. A. Quaranta, A review of some charge transport properties of silicon, Solid-State Electron., 1977, 20, 77-89.
170 W. Hong, Y. Xu, G. Lu, C. Li and G. Shi, Transparent graphene/PEDOT-PSS composite films as counter electrodes of dye-sensitized solar cells, Electrochem. Commun., 2008, 10, 1555-1558.

171 F. Li, K. Cai, S. Shen and S. Chen, Preparation and thermoelectric properties of reduced graphene oxide/PEDOT: PSS composite films, Synth. Met., 2014, 197, 58-61.

172 K. Zhang, Y. Zhang and S. Wang, Enhancing thermoelectric properties of organic composites through hierarchical nanostructures, Sci. Rep., 2013, 3, 3448.

173 Z.-H. Ge, Y. Chang, F. Li, J.-T. Luo and P. Fan, Boosted Thermoelectric Properties of PEDOT:PSS Polymer Bulk Prepared by Spark Plasma Sintering, Chem. Commun., 2018, 54(19), 2429-2431.

174 K. C. See, J. P. Feser, C. E. Chen, A. Majumdar, J. J. Urban and R. A. Segalman, Water-Processable Polymer? Nanocrystal Hybrids for Thermoelectrics, Nano Lett., 2010, 10, 4664-4667.

175 X. Cheng, L. Wang, X. Wang and G. Chen, Flexible films of poly(3,4-ethylenedioxythiophene):Poly(styrenesulfonate)/ SnS nanobelt thermoelectric composites, Compos. Sci. Technol., 2018, 155, 247-251.

176 X. Wang, F. Meng, Q. Jiang, W. Zhou, F. Jiang, T. Wang, X. Li, S. Li, Y. Lin and J. Xu, A Simple Layer-by-Layer Assembly Method for Simultaneously Enhanced Electrical Conductivity and Thermopower of PEDOT:PSS/ce-MoS2 Heterostructure Films, ACS Appl. Energy Mater., 2018, 1(7), 3123-3133.

177 X. Guan, W. Feng, X. Wang, R. Venkatesh and J. Ouyang, Significant Enhancement in the Seebeck Coefficient and Power Factor of p-Type Poly (3, 4-ethylenedioxythiophene): Poly (styrenesulfonate) through the Incorporation of n-Type MXene, ACS Appl. Mater. Interfaces, 2020, 12, 13013-13020.

178 H. Song, Q. Yang, W. Yao, K. Cai and J. He, Polymer/carbon nanotube composite materials for flexible thermoelectric power generator, Compos. Sci. Technol., 2017, 153.

179 H. Song, C. Liu, J. Xu, Q. Jiang and H. Shi, Fabrication of a layered nanostructure PEDOT:PSS/SWCNTs composite and its thermoelectric performance, RSC Adv., 2013, 3, 22065.

180 D. Kim, Y. Kim, K. Choi, J. C. Grunlan and C. Yu, Improved Thermoelectric Behavior of Nanotube-Filled Polymer Composites with Poly(3,4-ethylenedioxythiophene) Poly(styrenesulfonate), ACS Nano, 2010, 4, 513-523.

181 Q. Jiang, X. Lan, C. Liu, H. Shi, Z. Zhu, F. Zhao, J. Xu and F. Jiang, High-performance hybrid organic thermoelectric SWNTs/PEDOT:PSS thin-films for energy harvesting, Mater. Chem. Front., 2017, 2(4), 679-685.

182 D. Yoo, J. Kim, S. H. Lee, W. Cho, H. H. Choi, F. S. Kim and J. H. Kim, Effects of one- and two-dimensional carbon hybridization of PEDOT:PSS on the power factor of polymer thermoelectric energy conversion devices, J. Mater. Chem. A, 2015, 3, 6526-6533.

$183 \mathrm{~S}$. Liu, H. Li and C. He, Simultaneous enhancement of electrical conductivity and seebeck coefficient in organic thermoelectric SWNT/PEDOT: PSS nanocomposites, Carbon, 2019, 149, 25-32. 
184 F. Li, K. Cai, S. Shen and S. Chen, Preparation and thermoelectric properties of reduced graphene oxide/PEDOT:PSS composite films, Synth. Met., 2014, 197, 58-61.

185 J. Choi, J. Y. Lee, S. S. Lee, C. R. Park and H. Kim, HighPerformance Thermoelectric Paper Based on Double Carrier-Filtering Processes at Nanowire Heterojunctions, Adv. Energy Mater., 2016, 6(9), 1502181, DOI: 10.1002/ aenm.201502181.

186 E. J. Bae, Y. H. Kang, C. Lee and S. Y. Cho, Engineered nanocarbon mixing for enhancing the thermoelectric properties of a telluride-PEDOT: PSS nanocomposite, J. Mater. Chem. A, 2017, 5, 17867-17873.

187 Q. Meng, K. Cai, Y. Du and L. Chen, Preparation and thermoelectric properties of SWCNT/PEDOT:PSS coated tellurium nanorod composite films, J. Alloys Compd., 2018, 778.

188 X. Li, L. Liang, M. Yang, G. Chen and C.-Y. Guo, Poly(3,4ethylenedioxythiophene)/graphene/carbon nanotube ternary composites with improved thermoelectric performance, Org. Electron., 2016, 38, 200-204.

189 J.-H. Bahk, H. Fang, K. Yazawa and A. Shakouri, Flexible thermoelectric materials and device optimization for wearable energy harvesting, J. Mater. Chem. C, 2015, 3, 10362-10374.
190 S. J. Kim, J. H. We and B. J. Cho, A wearable thermoelectric generator fabricated on a glass fabric, Energy Environ. Sci., 2014, 7, 1959-1965.

191 M. Hyland, H. Hunter, J. Liu, E. Veety and D. Vashaee, Wearable thermoelectric generators for human body heat harvesting, Appl. Energy, 2016, 182, 518-524.

192 M. Zhang and J. T. W. Yeow, A flexible, scalable, and selfpowered mid-infrared detector based on transparent PEDOT: PSS/graphene composite, Carbon, 2019, 156, 339-345.

193 F. Zhang, Y. Zang, D. Huang, C. A. Di and D. Zhu, Flexible and self-powered temperature-pressure dual-parameter sensors using microstructure-frame-supported organic thermoelectric materials, Nat. Commun., 2015, 6, 8356.

194 J. Oh, J.-H. Kim, K. T. Park, K. Jo, J.-C. Lee, H. Kim and J. G. Son, Coaxial struts and microfractured structures of compressible thermoelectric foams for self-powered pressure sensors, Nanoscale, 2018, 10, 18370-18377.

195 L. Ruan, Y. Zhao, Z. Chen, W. Zeng, S. Wang, D. Liang and J. Zhao, A Self-Powered Flexible Thermoelectric Sensor and Its Application on the Basis of the Hollow PEDOT: PSS Fiber, Polymers, 2020, 12, 553. 